BMC

Evolutionary Biology

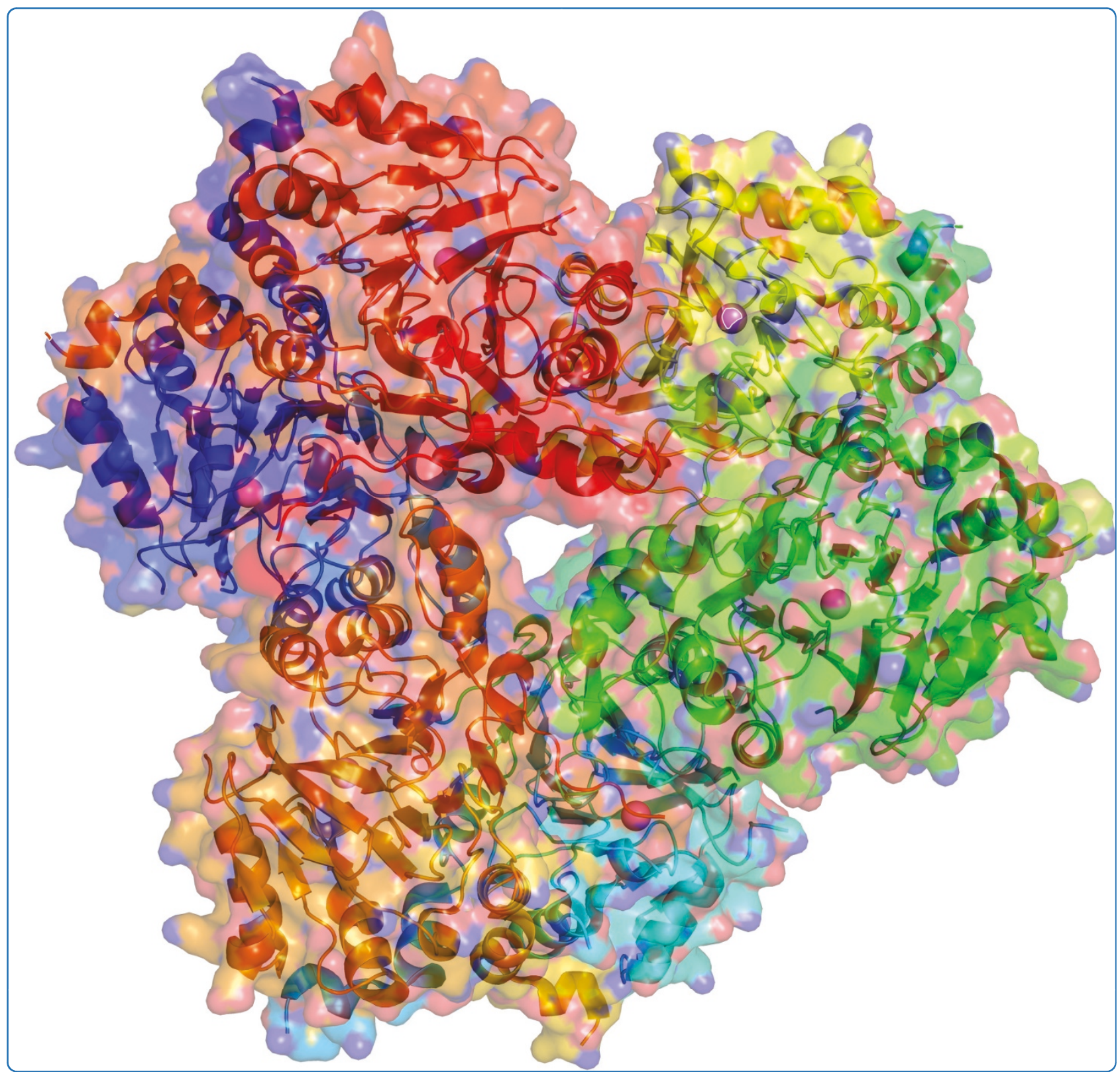

\title{
The UlaG protein family defines novel structural and functional motifs grafted on an ancient RNase fold
}

Fernandez et al. 


\title{
The UlaG protein family defines novel structural and functional motifs grafted on an ancient RNase fold
}

Francisco J Fernandez ${ }^{1 *}$, Fernando Garces ${ }^{2}$, Miguel López-Estepa ${ }^{1}$, Juan Aguilar ${ }^{3}$, Laura Baldomà ${ }^{3}$, Miquel Coll ${ }^{4,5}$, Josefa Badia ${ }^{3}$ and M Cristina Vega ${ }^{*}$

\begin{abstract}
Background: Bacterial populations are highly successful at colonizing new habitats and adapting to changing environmental conditions, partly due to their capacity to evolve novel virulence and metabolic pathways in response to stress conditions and to shuffle them by horizontal gene transfer (HGT). A common theme in the evolution of new functions consists of gene duplication followed by functional divergence. UlaG, a unique manganese-dependent metallo- $\beta$-lactamase (MBL) enzyme involved in L-ascorbate metabolism by commensal and symbiotic enterobacteria, provides a model for the study of the emergence of new catalytic activities from the modification of an ancient fold. Furthermore, UlaG is the founding member of the so-called UlaG-like (UlaGL) protein family, a recently established and poorly characterized family comprising divalent (and perhaps trivalent) metal-binding MBLs that catalyze transformations on phosphorylated sugars and nucleotides.
\end{abstract}

Results: Here we combined protein structure-guided and sequence-only molecular phylogenetic analyses to dissect the molecular evolution of UlaG and to study its phylogenomic distribution, its relatedness with present-day UlaGL protein sequences and functional conservation. Phylogenetic analyses indicate that UlaGL sequences are present in Bacteria and Archaea, with bona fide orthologs found mainly in mammalian and plant-associated Gramnegative and Gram-positive bacteria. The incongruence between the UlaGL tree and known species trees indicates exchange by HGT and suggests that the UlaGL-encoding genes provided a growth advantage under changing conditions. Our search for more distantly related protein sequences aided by structural homology has uncovered that UlaGL sequences have a common evolutionary origin with present-day RNA processing and metabolizing MBL enzymes widespread in Bacteria, Archaea, and Eukarya. This observation suggests an ancient origin for the UlaGL family within the broader trunk of the MBL superfamily by duplication, neofunctionalization and fixation.

Conclusions: Our results suggest that the forerunner of UlaG was present as an RNA metabolizing enzyme in the last common ancestor, and that the modern descendants of that ancestral gene have a wide phylogenetic distribution and functional roles. We propose that the UlaGL family evolved new metabolic roles among bacterial and possibly archeal phyla in the setting of a close association with metazoans, such as in the mammalian gastrointestinal tract or in animal and plant pathogens, as well as in environmental settings. Accordingly, the major evolutionary forces shaping the UlaGL family include vertical inheritance and lineage-specific duplication and acquisition of novel metabolic functions, followed by HGT and numerous lineage-specific gene loss events.

\footnotetext{
* Correspondence: franfdez@cib.csic.es; cvega@cib.csic.es

${ }^{1}$ Structural and Quantitative Biology Department, Centro de Investigaciones

Biológicas (CIB-CSIC), Ramiro de Maeztu 9, 28040 Madrid, Spain

Full list of author information is available at the end of the article
} 


\section{Background}

Bacteria can evolve complex virulence mechanisms and metabolic pathways by gene duplication and functional divergence of one of the copies [1,2], with the ancestral copy retaining the original function, and by shuffling these entire pathways by horizontal gene transfer (HGT) $[3,4]$. Indeed, gene duplication has been presented as a major driving force behind evolutionary change [1,2], with $70 \%$ and $90 \%$ of duplicated domains found in bacteria and eukaryotic organisms, respectively [5]. However, the relative impact of gene duplication and HGT in the generation of genetic diversity and their role in adaptation is still under debate [3]. The opposite process of gene loss has been hypothesized to favor the evolution of prokaryotes under intermittent selective pressures (coupled to the regain of function in the form of cryptic genes, such as those involved in $\beta$-glucoside degradation found in most natural isolates of $E$. coli) [6,7]. Other examples of gene loss occur in response to lifestyle adaptations like the dramatic genome reduction of obligate host-associated bacteria such as Mycoplasma and Buchnera [8], or to allow for more efficient replication in free-living microorganisms like in the marine free-living bacteria Prochlorococcus and Pelagibacter $[9,10]$. The prevalence of HGT in bacteria, especially in multi-species ecological communities such as those in the human gastrointestinal tract, plays an essential role in generating genetic variation in addition to gene duplication and gene loss [11]. HGT events are crucial for the dissemination of beneficial functional traits between bacterial communities especially when these are under strong selection pressure, as is the case for virulence factors among pathogenic bacteria $[12,13]$.

A number of protein folds have been extensively re-used during evolution for the emergence of novel functions, including metallo- $\beta$-lactamase (MBL) [14], triose-phosphate isomerase (TIM) [15,16], and Rossmann fold scaffolds [15], in particular in the context of metabolism, which plays a crucial role in bacterial adaptation as shown by the massive expansion of metabolic superfamilies since the last universal common ancestor (LUCA) [17]. One of the underlying reasons for the success of these folds in acquiring new functions is that they hold a wide active site that can accommodate complex and multiple substrates simultaneously and the presence of many surrounding loops, modifications of which can alter the chemical and catalytic properties of the protein, its substrate/product profile, or cofactor-binding properties. MBLs provide an important source of versatile catalysts that have an ancient evolutionary origin and a widespread phylogenomic distribution across Bacteria, Archaea, and Eukarya [14]. The central role of MBLs in both cellular and metabolic housekeeping functions as well as in the rapid spread of multidrug resistant pathogens calls for a better understanding of the evolutionary mechanisms by which novel functions evolve from an archetypical protein fold.

UlaG, a recently characterized MBL sequence with a novel function and unique sequence and structural features $[18,19]$, is a protein encoded by a gene from the L-ascorbate utilization ( $u l a$ ) regulon of Enterobacteria [20]. The ula regulon consists of two divergently transcribed units (Additional file 1). The first is the ulaG cistron that encodes the L-ascorbate 6-phosphate lactonase (UlaG) involved in the conversion of L-ascorbate 6phosphate to L-gulonate 6-phosphate in the catabolism of L-ascorbate [18] (Additional file 2). The second unit comprises the ulaABCDEF operon. Genes ulaABC encode the transmembrane phosphotransferase system (PTS) responsible for the uptake of L-ascorbate from the medium in the form of L-ascorbate 6-phosphate. The structural genes ula $\mathrm{D}$ (3-keto-L-gulonate 6-phosphate decarboxylase), ulaE (L-xylulose 5-phosphate 3-epimerase), and ulaF (L-ribulose 5-phosphate 4-epimerase), act sequentially on the product of UlaG to convert it to Dxylulose 5-phosphate, an intermediate of the pentose phosphate pathway (Additional file 2). The ula regulon is under transcriptional control of ulaR, located upstream to $u l a \mathrm{G}$. Expression of the structural genes of the ula regulon is controlled by changes in the quaternary architecture of UlaR, a DeoR-family repressor, which, in the absence of extracellular L-ascorbate, represses transcription of the regulon by binding DNA as a homotetrameric complex to four operator sequences (Additional file 1). The availability of L-ascorbate triggers transcriptional activation by binding of the inducer molecule $\mathrm{L}$-ascorbate 6-phosphate to UlaR, thereby leading to its concomitant conversion to a DNA-free dimeric complex that can no longer maintain a repressed state [21].

Of the L-ascorbate catabolic genes, only the catalytic activity of the ula $\mathrm{G}$ gene product has not been confidently assigned on the basis of sequence homology, and it was not until last year [18] that the activity of UlaG was established experimentally. In addition, the determination of the crystal structure of UlaG at 2.6- $\AA$ resolution [18] corroborated sequence-based predictions that the fold was similar to $\mathrm{Zn}^{2+}$-dependent hydrolases of the MBL superfamily while simultaneously revealing several unforeseen properties (Figure 1A-E). First, the UlaG fold resembles that of MBL RNases with additional conspicuous adaptations to its new functional role in L-ascorbate catabolism (Figure $1 \mathrm{~B}$ and $1 \mathrm{C}$ ). Second, it is the first characterized $\mathrm{Mn}^{2+}$-dependent MBL (Figure 1D). And, third, UlaG has a distinctive oligomeric structure (a hexamer) that had not been previously described for MBLs (Figure 1E). Prompted by these unusual properties of $\mathrm{UlaG}$, which suggested that the ancestral RNase fold of this protein had been subjected to extensive modifications in order to acquire new 


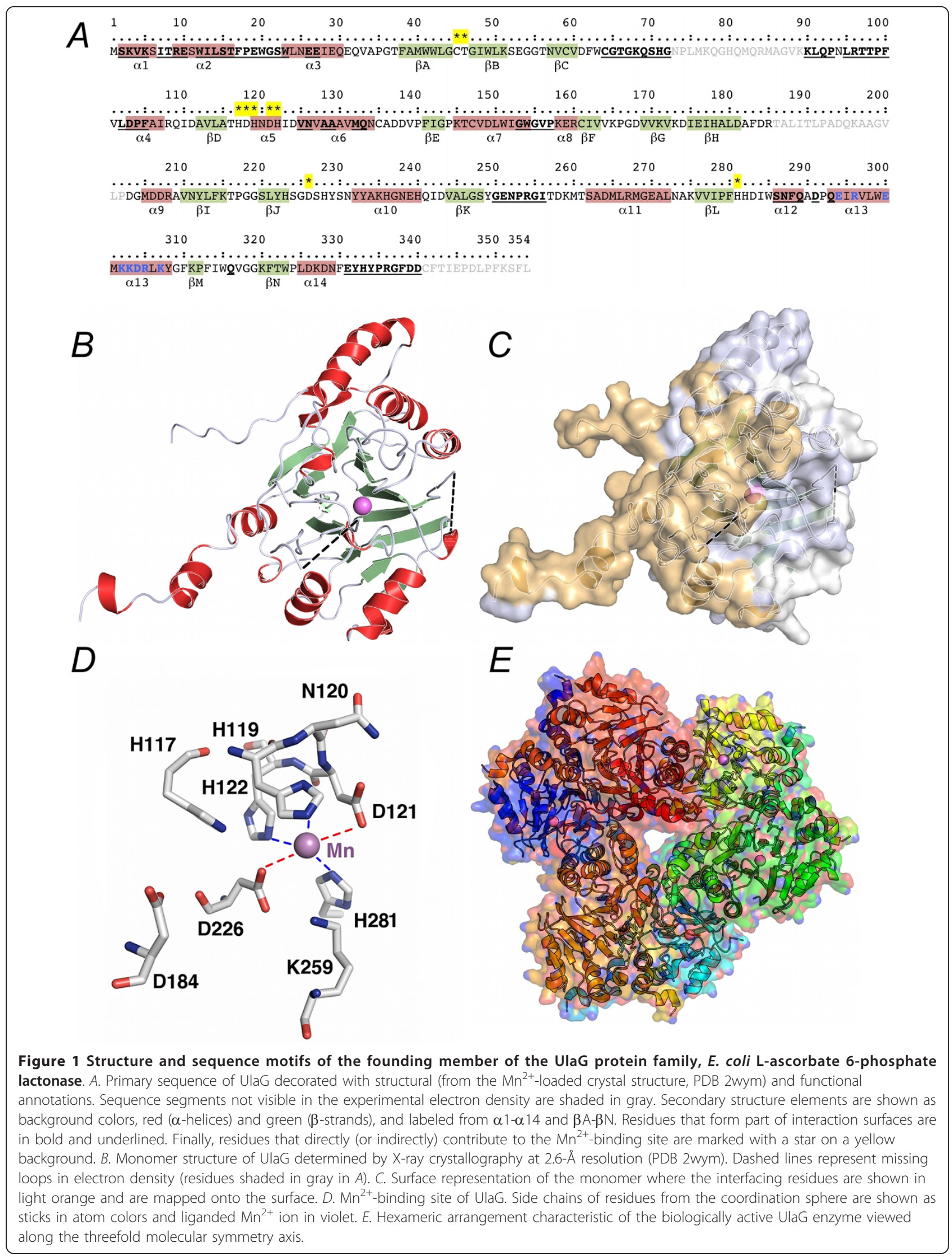


catalytic activity and regulatory mechanisms in a completely different ecological scenario [15], we set out an indepth study to characterize the protein family founded by UlaG. In this study we present the molecular evolution of the UlaG protein family, its phylogenomic distribution, and genome context, and we construct an evolutionary pathway from the ancestral RNase fold to present-day UlaG-like proteins in prokaryotic organisms.

\section{Results}

\section{Phylogenetic analysis}

Sequence similarity searches with E. coli UlaG primary sequence [Genbank:P39300] against a database of nonredundant protein sequences revealed that proteins with sequence identity greater than $50 \%$, and therefore possibly homologous to UlaG, are present in bacterial genomes belonging to three eubacterial phyla across several classes and families (Figure 2; Additional files 3 and 4). Bacterial lineages containing at least one potential UlaG homolog, or UlaG-like (UlaGL) sequence, include families from the divisions Proteobacteria (Enterobacteriaceae genera Escherichia, Salmonella, Shigella, Citrobacter, Enterobacter, Klebsiella, Yersinia, Providencia, Actinobacillus, Haemophilus, Mannheimia, and Pasteurella; Vibrionales genera Vibrio and Photobacterium); Firmicutes (Clostridiales genus Clostridium; Ruminococcaceae genera Anaerotruncus, Ruminococcus, and Epulopiscium; Lactobacillales genera Enterococcus, Lactobacillus, Leuconostoc, and Streptococcus) and Actinobacteria (Coriobacteriaceae Atopobium). These genera include commensal and symbiotic bacteria, opportunistic pathogens, and pathogenic bacteria that colonize the mammalian gastrointestinal tract (GIT), oral mucosa, genitourinary tract, as well as bacteria found in environmental reservoirs such as soil and water.

Comparison of unrooted phylogenetic trees constructed from curated multiple sequence alignments of UlaGL sequences (Figure 3; Additional files 5, 6 and 7) with the known phylogeny of Proteobacteria and Grampositive bacteria [22-24] allows us to tentatively place

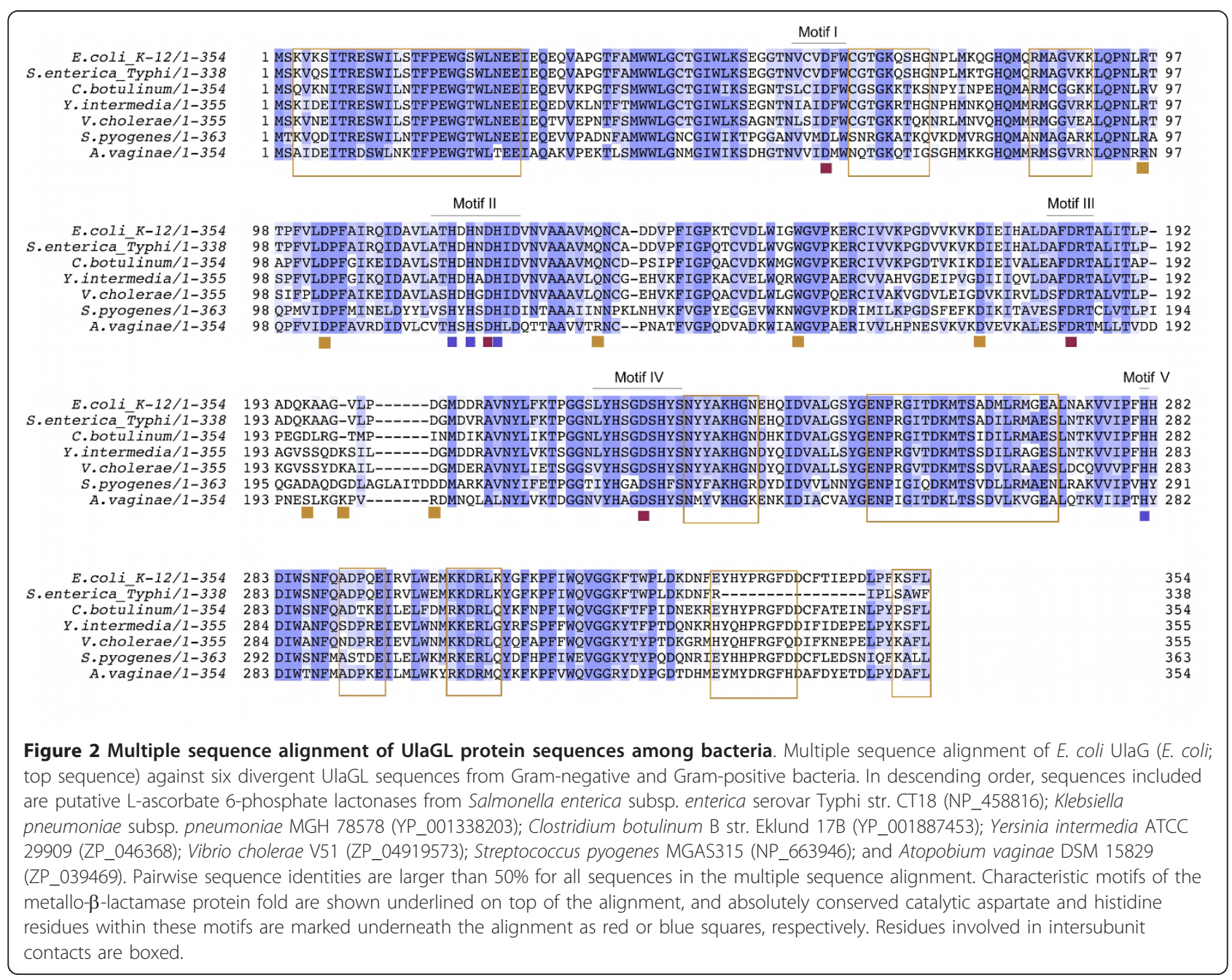




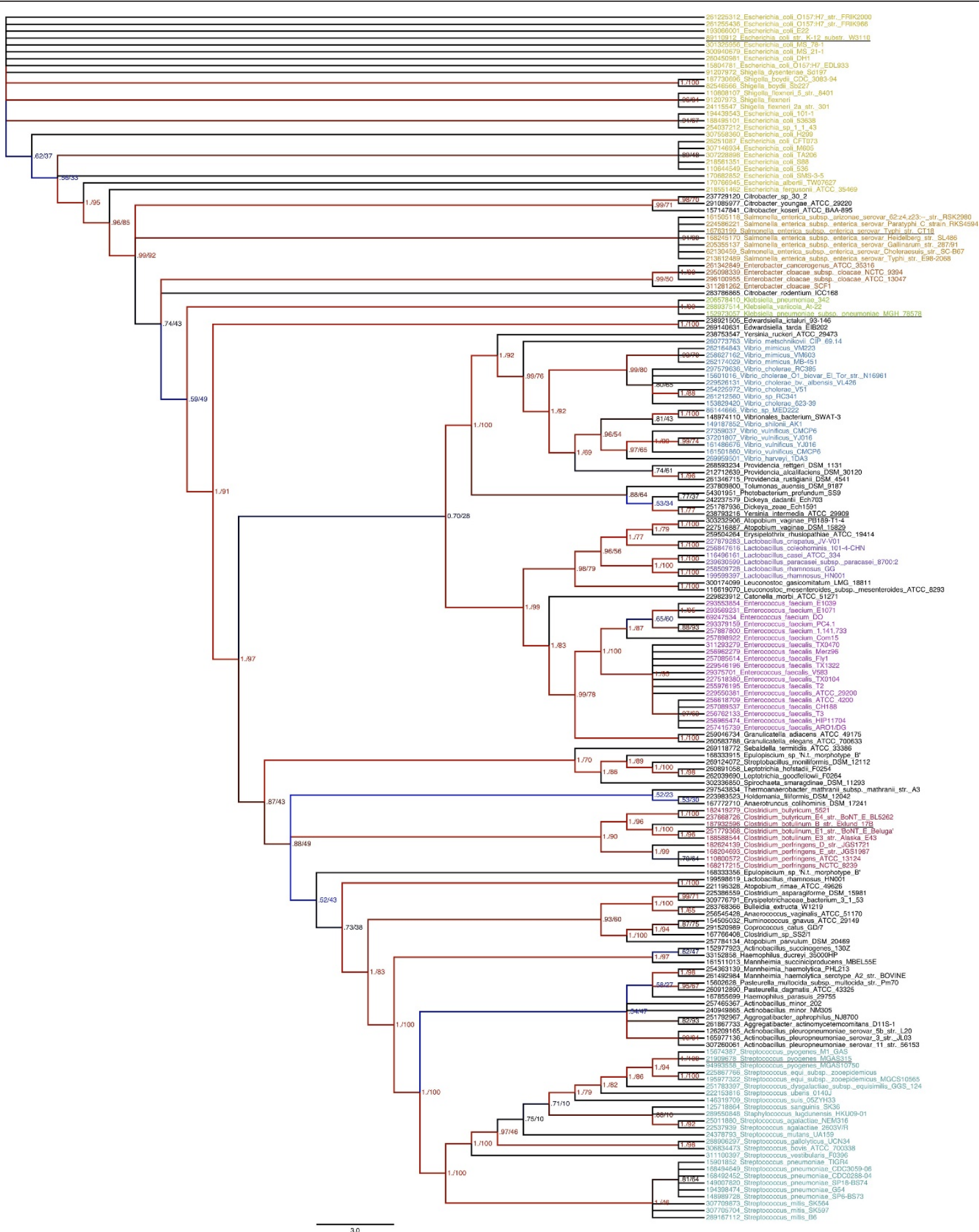

Figure 3 Bayesian inference tree of UlaGL orthologs from 330 aligned amino acid positions. The consensus tree was produced with FigTree from the last 9,500 trees representing 950,000 stationary generations. Bayesian inference posterior probability (BIPP) and maximumlikelihood bootstrap percentage (MLBP) are indicated on nodes. Only branches with $>0.50$ BIPP support are labeled with BIPP and MLBP. The SDSF at the end of the MrBayes run was 0.008 . The scale bar below the tree shows the evolutionary distance expressed as substitutions per site. Taxon names are prefixed with their NCBI GI numbers. The underlined taxa are shown in the representative alignment (Figure 2). Nexus alignment files are available from the authors on request. 
the last common ancestor around the radiation of the Firmicutes from the common branch that would later split into Proteobacteria and Actinobacteria. Interestingly, the first radiation event in the phylogenetic tree of the UlaG homologs coincides with the emergence of class B3 $\beta$-lactamases, prior to the split of Proteobacteria from Gram-positive bacteria [25]. This observation is consistent with the proposed evolutionary origin of MBL sequences without $\beta$-lactamase activity from an ancient, essential protein, such as an RNA-processing enzyme [14,25].

The analysis of the UlaGL gammaproteobacterial subtree (a tree containing solely gammaproteobacterial sequences) and its comparison to the corresponding species tree (Additional file 8) using the Shimodaira-Hasegawa test supports a model of near exclusive vertical transmission ( $p=1.0$ at $5 \%$ confidence level). In contrast, neither the Firmicutes nor the Actinobacteria branches are completely orthologous groups. The Actinobacteria, represented by Atopobium spp., appears interspersed between proteobacterial and Firmicutes lineages, while the Firmicutes branches contain sequences from enterobacterial species (e.g., Yersinia spp., Dickeya spp., and Mannheimia spp.). The incongruent placement of enterobacterial sequences within Gram-positive clades indicates that some HGT events between enterobacteria and bacteria from other taxonomic groups within these categories have occurred. This possibility has already been proposed for the MBL superfamily [25]. These observations suggest a far more recent origin for ulaGL in the common ancestor to Vibrionales, Enterobacteriales and Pasteurellales. In this scenario, the absence of an UlaGL protein in distantly related Gammaproteobacteria (e.g., Pseudomonas, Xanthomonas or Xylella) would be a natural consequence of the ulaGL birth after the split of their common ancestor. In addition, the current distribution of ulaGL within Firmicutes/Actinobacteria would require only a very reduced number of HGT events with an enterobacterial species as donor and a Gram-positive bacterium as recipient. A case in point is the presence of Pasteurella and Mannheimia UlaGL nested inside the Clostridium and Streptococcus clades; this tree topology can be explained by one HGT event from a Pasteurelalles ancestor to the common ancestor of (Clostridiales + Streptococcales) or several later HGT events to each individual recipient lineage.

For most prokaryotic species, we have identified a single UlaG homolog per genome (Additional file 3). Indeed, we detected only 4 species with more than one UlaG homolog (above an E-value threshold of $1 \times 10^{-10}$ ). This finding thus indicates the existence of paralogous sequences in restricted cases. Three such species are eubacterial, including Epulopiscium sp. 'N.t. morphotype B' (2 paralogs), Dickeya zeae Ech159 (2 paralogs), and Sebaldella termitidis (2 paralogs), and one is archeal,
Haloterrigena turkmenica DSM 5511 (3 paralogs). In Epulopiscium sp. 'N.t. morphotype B' the two sequences (gi|168333356 and gi|168333915) are annotated as hypothetical proteins, and the first sequence lacks similarity to the first 18 amino acids of UlaG, thus the $\mathrm{N}$-terminal hexamerization motif has been weakened or abolished. The two paralogous sequences of Dickeya zeae Ech1591 (ref|YP_003003802.1 and gb|ACT06323.1) are also listed as hypothetical proteins. In contrast, the UlaGL paralogs in S. termitidis are annotated as L-ascorbate 6-phosphate lactonases and are part of a near complete operon duplication. Except for sequencing or annotation error, the two UlaGL sequences in S. termitidis share $100 \%$ sequence identity, which is consistent with a very recent duplication event.

Although RNA processing enzymes did not appear to have generally been captured by a sequence-only search, at least one such sequence was present in iteration 1 of PSI-BLAST. It corresponds to a putative mRNA 3'-end processing factor from the Crenarcheota Pyrobaculum islandicum DSM 4184. The length of the alignment is limited (about 100 residues) but in this limited length it contains $33 \%$ sequence identity and $48 \%$ similarity, and contains similarity to the amino-acid stretch spanning 96-182, a region that includes the central portion of the active site and adjacent elements. This degree of sequence conservation places this hit slightly above the twilight zone of hard-to-establish similarity, and, in conjunction with the structural homology to MBL RNases, provides further evidence of a distant evolutionary relation of the UlaG family with MBL RNases.

Finally, we detected a series of sequences corresponding to the $\operatorname{rom} A$ gene encoding for a multi-drug resistance membrane protein and bearing some degree of sequence homology to UlaG. However, the low sequence identity (always below the 30\% identity threshold; best hits had $18 \%$ identity, 35\% similarity, and 350-360 aligned residues, with a bit score of 327 and a E-value of $10^{-87}$ ) makes this link less reliable. However, it is not inconceivable that the UlaG and the RomA proteins had an ancient common ancestor and that their markedly different functions arose by divergence over a very long evolutionary time.

\section{Conservation of UlaG features among orthologous sequences}

UlaG has several distinctive motifs that confer its characteristic structure and support its catalytic activity and that are expected to be conserved among putative orthologous sequences. Aside from the metal-binding motif and the presence of long loops over the active site, presumably for substrate binding and assisting catalysis, which are also found in more distantly related MBLs, the most conspicuous features of UlaG are found in its amino $(\mathrm{N})$ and carboxy $(\mathrm{C})$ termini. The $\mathrm{N}$ terminus 
spans thirty residues and folds as three consecutive $\alpha$ helices (Figure 1A and 1B). Helices $\alpha 1$ and $\alpha 2$ form a mostly positively charged extended arm that inserts into a complementary, negatively charged valley made from helix $\alpha 3$, thereby suggesting that the conservation of this oligomerization motif requires the conservation of the complementary features of helices $\alpha 1-\alpha 2$ and $\alpha 3$. Truncations of the two first $\alpha$-helices $\alpha 1$ and $\alpha 2$ are expected to weaken the greatest contact area between adjacent UlaG subunits and, accordingly, would destabilize a putative hexameric complex. Likewise, though less dramatically, C-terminal truncations deleting residues 331-340, located immediately after the last $\alpha$-helix, $\alpha 14$, would be expected to remove contact area residues and therefore compromise the oligomeric structure. Indeed, of all the 180 bona fide UlaG orthologs only in Shigella dysenteriae Sd197 the N-terminal helices $\alpha 1-\alpha 2$ are lacking. The only other orthologous sequence with an incomplete $\mathrm{N}$ terminus, Mannheimia haemolytica PHL213 UlaG, appears to lack helix $\alpha 1$. However, it is uncertain whether the apparent loss is reliable or caused by a sequencing artifact. Interestingly, the presence of additional N-terminal residues is observed more often than the loss of the $\mathrm{N}$-terminal helical motif. In 53 out of 180 sequences (29.4\%), the $\mathrm{N}$ terminus contains additional residues when compared to the $E$. coli UlaG reference sequence. Of these, 15 sequences (28\%) have $\mathrm{N}$-terminal extensions longer than 5 residues (the longest having 31 amino acids) and most (14 or 92\%) have not lost a methionine residue at the reference position of Met-1. Since all these sequences are uncharacterized, it is still unclear whether the predicted initiation codon is used or whether the reference reading frame is used instead. In support of the correct assignment of the predicted initiation codons, virtually all these sequences contain 1-3 lysine residues, which might play a similar role to the two lysine residues in helix $\alpha 1$.

The C-terminal end of E. coli UlaG contains elements essential for the oligomerization and for organization of the channel-like architecture of the hexameric molecule (Figure 1, A, 1C and 1E). Residues 285-354 encompass helices $\alpha 12$ and $\alpha 13$, loop $\beta \mathrm{M}-\beta \mathrm{N}$, and a 10-amino-acid stretch following helix $\alpha 14$, all of which include interface residues. Helix $\alpha 13$ occupies a central location in the UlaG molecule and is hypothesized to provide further stabilization to the quaternary structure by the interactions it makes with the symmetrical helices in the other subunits. The crucial role of the $\mathrm{C}$ terminus is underlined by the fact that only three sequences among the orthologous set show considerable deviations from the consensus sequence. Two of these sequences (cattle pathogenic Escherichia coli O157:H7 str. FRIK2000 and FRIK966) lack a stretch from helix $\alpha 14$ to the $C$ terminus, apparently as a result of the mutation of a tryptophan TGG codon (Trp-323 in E. coli UlaG) to a stop TAG codon. The third sequence, from Salmonella enterica subsp. enterica serovar Typhi str. E98-2068, has specifically lost the ten amino-acid residues involved in intersubunit contacts while it has retained helix $\alpha 14$ as well as the last 7 amino acids.

\section{Highest variable regions among closely related sequences} Among highly similar regions, for example, across different $E$. coli strains, single amino-acid substitutions tend to be conservative substitutions of small hydrophobic or polar uncharged residues in or preceding $\beta$-strands, including positions in the neighborhood of the active site. This observation is not surprising since small hydrophobic residues in $\beta$-strands frequently have structural support roles that do not cause changes in function. As for $\alpha$ helices, for example, Met-301 in $\alpha 13$ is conservatively replaced by an isoleucine residue in Shigella flexneri UlaG, where it is the only amino-acid change along the entire length of the sequence. In this particular case, the side chain of Met-301 points to the solvent channel in the middle of the UlaG hexamer and is unlikely to carry any critical functional role in stability or catalysis.

Inspection of hyper-variable regions between closely related enterobacterial sequences shows that most of the variation does cluster in two $\alpha$-helices, $\alpha 5$ (spanning residues 145-154) and $\alpha 7$ (262-271). In both cases, the mutations are conservative and should not disrupt the structural elements surrounding them. The $\alpha 5$ helix is fully exposed and its $C$ terminus establishes helix-helix dipolar interactions with the short $\alpha 1$ helix. In contrast, $\alpha 7$ is part of the second largest interface in the quaternary structure of UlaG, where it forms part of two symmetrical $\alpha$-helical bundles with an extensive surface interaction.

As soon as sequence identity falls below $80 \%$, substitutions appear in and around the active site and metalbinding loops, suggesting that there might be shifts in substrate specificity or reactivity. In UlaG homologous sequences found in various Clostridium sp. including human pathogenic strains with less than $80 \%$ sequence identity (74-76\%), all metal-binding ligands are conserved against a background of mostly conservative, evenly distributed mutations. This indicates that the overall structure has been preserved despite the possibility that the active site loops accumulated enough changes to have shifted in substrate specificity.

\section{Phylogenetic analysis of prokaryotic halophilic UlaGL}

The closest non-eubacterial UlaGL sequences are present in Archaea from Euryarcheota and display 26\% sequence identity ( $42 \%$ similarity) with the top aligned UlaGL sequences (Figure 4A; Additional files 9, 10 and 11). These archeal sequences appear to be restricted to 

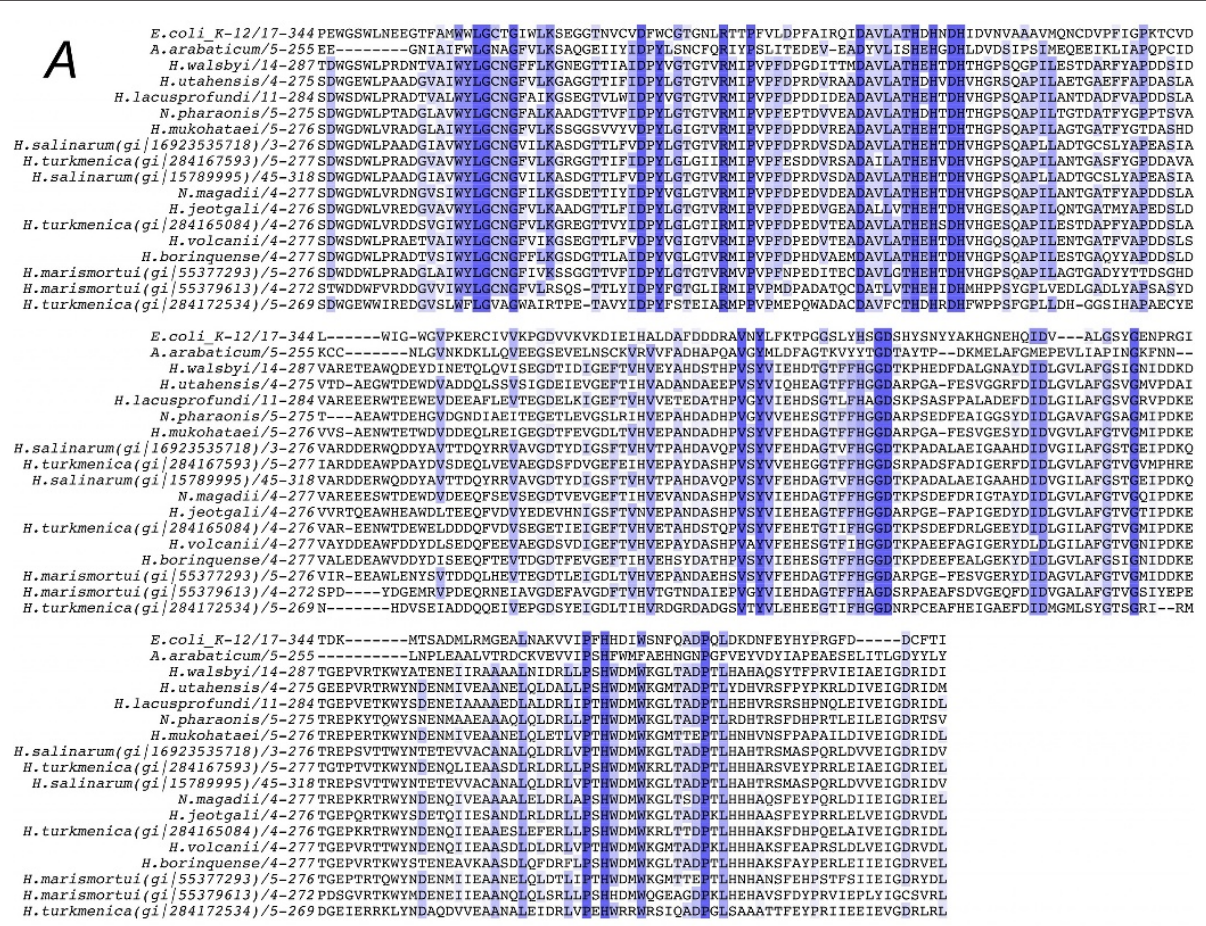

\section{$B$}

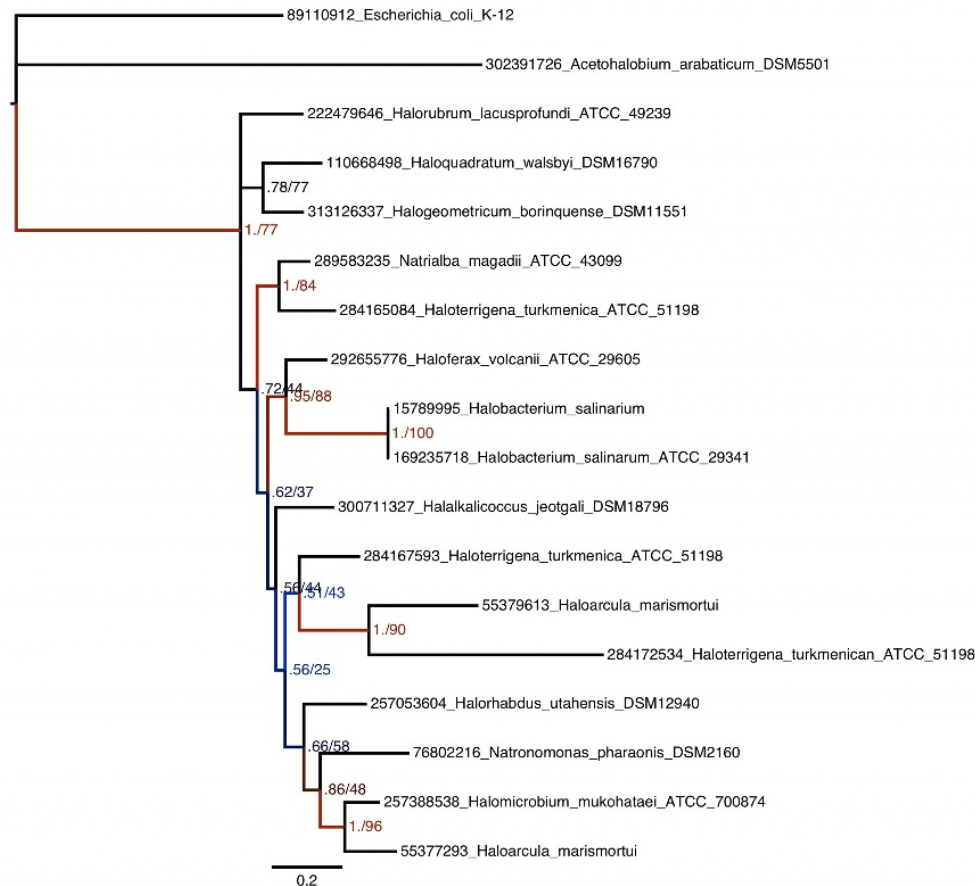

Figure 4 Horizontal gene transfer of UlaGL genes to halophilic Archea. A. Alignment of UlaGL sequences from halophilic archea, along with two reference sequences (E. coli UlaG, and the halophilic bacterium Acetohalobium arabaticum). B. Bayesian consensus tree of the same sequences in $A$, with E. coli UlaG as the outgroup. Bayesian inference posterior probability (BIPP) and maximum-likelihood bootstrap percentage (MLBP) are indicated on nodes. Only branches with > 0.50 BIPP support are labeled with BIPP and MLBP. The SDSF at the end of the MrBayes run was 0.007 . The scale bar below the tree shows the evolutionary distance expressed as substitutions per site. Taxon names are prefixed with their NCBI GI numbers. Nexus alignment file is available from the authors on request. 
several extremophilic species of the Halobacteriaceae family (Figure 4), including organisms such as Haloterrigena turkmenica DSM 5511, Haloquadratum walsbyi DSM 16790, Halorhabdus utahensis DSM 12940, Natronomonas pharaonis DSM 2160, Halogeometricum borinquense DSM 11551, Halobacterium sp. NRC-1, Halobacterium salinarum R1, Haloferax volcanii DS2, Halomicrobium mukohataei DSM12286, Natrialba magadii ATCC 43099, Halorubrum lacusprofundi ATCC 49239, Halalkalicoccus jeotgali B3, and Haloarcula marismortui ATCC 43049. This severely restricted ecological distribution of the archeal UlaGL sequences suggests that the most parsimonious evolutionary scenario for their origin is an HGT event from a eubacterial species. In support of this hypothesis, the eubacterial halobacterium Acetohalobium arabaticum DSM 5501, which thrives under similar environmental conditions as the Halobacteriaceae, shares with this family the same protein sequence, which shows a similar degree of conservation with the cluster of UlaGL sequences (24-26\%). Furthermore, phylogenetic trees reconstructed from alignments of the archeal sequences and the E. coli and A. arabaticum UlaGL sequences provide support for a tight clustering in the origin and diversification of the halophilic archeal UlaGL sequences, with multiple duplication events in various lineages (including Haloterrigena turkmenica, Halobacterium salinarum, and Haloharcula marismortui) (Figure 4B; Additional file 12). In agreement with the proposal that these archeal UlaGL sequences are functionally unrelated to the remaining eubacterial UlaGLs, they all conserve a core sequence spanning approximately 275 residues that lacks two critical regions for UlaG oligomerization, the $\mathrm{N}$-terminal $\alpha 1-\alpha 2$ motif and $\alpha 13$ (the central channel helix) and $\beta M$ $\beta \mathrm{N}$, as well as 20 -amino-acid segment of the loop $\beta C-\beta 4$ following catalytic motif I (Figure 1 and $4 \mathrm{~A}$ ). The absence of the extreme $\mathrm{N}$ - and $\mathrm{C}$-terminal sequences that are involved in shaping the characteristic hexameric structure of UlaG, suggests that the haloextremophile sequences, both archeal and eubacterial, do not assemble into hexameric structures.

\section{Conservation of the ula regulon structure and genomic context}

It has been proposed that the organization of genes into operons is the main reason for conservation of adjacency in prokaryotic genomes [26]. The genomic context can provide insight into function, since functionally related genes tend to be contiguous and co-transcribed in bacteria [22]. Accordingly, we searched the genomes that encode proteins with significant sequence identity to UlaG (putative orthologs, with sequence identity greater than 55\%) to study whether the homologous gene was part of a similar regulon structure to that found in E. coli.
As a straightforward method, we first searched for UlaR homologous proteins whose respective genes would be adjacent to those encoding the corresponding UlaG homologs. Indeed, a ulaR homologous gene was found adjacent to each ula G homologous gene in all Gramnegative and Gram-positive genomes examined except for the phylogenetically-isolated Fusobacterium Sebaldella termitidis ATCC 33386 [27] and Streptococcus spp., where a gene encoding a BglG transcription regulator was found next to the respective ula $\mathrm{G}$ instead of ula $\mathrm{R}$ encoding a DeoR-family repressor (Figure 5). Proteins belonging to the BglG family are bacterial RNA-binding regulatory proteins that control the expression of genes and operons required for the utilization of specific carbohydrates [28]. Furthermore, the ulaABCDEF unit was found in all cases in spite of the observation of various species-specific gene rearrangements, insertions and losses (Figure 5). These modifications, together with sequence divergence between the orthologous pair genes, cause the exact nature of the metabolic pathway catalyzed by the homologous ulaDEF units to become blurred with evolutionary divergence. The greatest conservation in operon structure and genomic context was found among the mammalian-associated Enterobacteria, both in the symbiotic intestinal microbiota as in human pathogens such as Klebsiella pneumoniae and Salmonella enterica serovar Typhi, with the E. coli ula regulon representing the reference sequence. Thus, the type ula regulon (Figure 5; Additional file 1) contains adjacent ula $\mathrm{R}$ and $u l a \mathrm{G}$ genes and the ulaABCDEF operon, which is transcribed from the opposite strand. This cluster is flanked on its 5 ' end by hypothetical proteins of the CP457 prophage region and on its 3' end by genes encoding proteins of unknown function (e.g., DUF1471) and by the genes coding for ribosomal subunit proteins (e.g., rpsF, for the $30 \mathrm{~S}$ ribosomal subunit protein S6). In closely related Enterobacteria some of these contiguous genes are transcribed in the same direction as ula $\mathrm{G}$ or ula$\mathrm{ABCDEF}$, with no intervening gene on the complementary strand, and they are conserved in multiple species (Figure 5, Additional file 1). These genes encode proteins with various functions but their physical proximity does not appear to bear any functional relevance to the utilization of L-ascorbate 6-phosphate. Among the enterobacterial genomes analyzed, only Edwardsiella ictaluri breaks this trend with its ula regulon flanked by genes encoding a hypothetical membrane protein and an $n f u A-$ encoding $\mathrm{Fe} / \mathrm{S}$ biogenesis protein.

A second group with a conserved operon structure includes genomes of Gram-negative Proteobacteria with diverse lifestyles, such as the environmental Aeromonadales bacterium Tolumonas auensis, the deep-sea Photobacterium profundum, the plant pathogen Dickeya zeae (the causative agent of soft rot in maize), and the human 


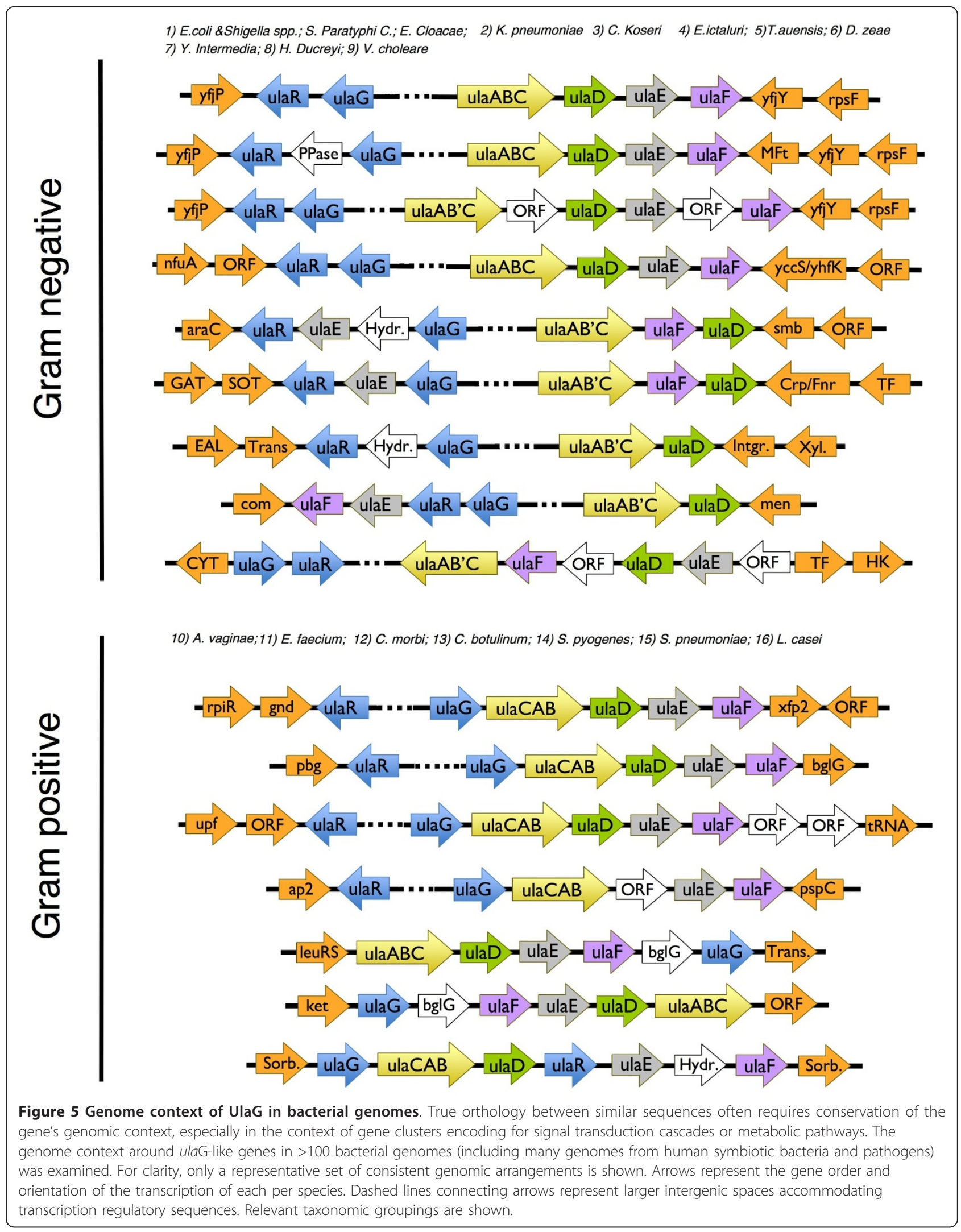


pathogens Yersinia spp., Haemophilus spp., and Vibrio cholerae. These microorganisms possess ula regulons that share various characteristics, such as a two-subunit PTS transporter wherein the large subunit (UlaAB)encoding gene is equivalent to a gene fusion of ula $\mathrm{A}$ and ula $\mathrm{B}$ (Figure 5). In this group it is common to observe a gene rearrangement whereby the ula $\mathrm{E}$ or $u l a \mathrm{~F}$ genes (or both) are inserted between or next to the ulaR and ulaG cistrons (e.g., H. ducreyi); insertions of an unrelated gene encoding a Cof-like hydrolase are also common (e.g., $Y$. intermedia). Since these additional genes are to be cotranscribed from a different transcriptional unit than the remaining structural genes, their regulatory control might become less constrained and therefore freer to be fine-tuned in response to the metabolic pressures of the bacterium. Another shared feature is the completely unrelated genomic contexts among all these bacteria, although their ula regulons tend to be bracketed by genes encoding enzymes involved in sugar metabolism (e.g., transaldolases and transketolases in T. auensis, amylose in $V$. cholerae), redox cofactor biosynthesis (e.g., menaquinone and phylloquinone in $H$. ducreyi), and transcription factors (e.g., D. zeae, V. cholera).

In $Y$. intermedia, the ula regulon is flanked on either side by what appears to be $\mathrm{N}$-terminally truncated genes corresponding to a transposase B (65 residues)/integrase (44 residues) enzyme pair; in $Y$. enterocolitica, the corresponding orthologs have been conserved in full length, and for example $Y$. enterocolitica integrase gene encodes a 450 -amino-acid polypeptide that has $97 \%$ identity with the 44-residue (predicted) polypeptide in Y. intermedia. The flanking transposase/integrase cassette suggests that the ula regulon is part of a still mobile metabolic island (or islet) in $Y$. enterocolitica [4], whereas in $Y$. intermedia the mobility may have been abrogated by partial gene deletion. In addition, the finding of a syntenic association between the ula regulon and a nearby tRNA gene (yinte0001_t290) in $Y$. intermedia lends further support to the hypothesis that the $Y$. intermedia ula regulon belongs to an ancient mobile DNA segment [4].

While in Gram-negative bacteria the ulaR/ulaG cistrons are transcribed co-directionally, in all Gram-positive bacteria that we have analyzed one of the following applies: (1) ulaR and ulaG are transcribed divergently, with ulaG being the first structural gene upstream of the PTS transporter genes (e.g., Atopobium vaginae, Enterococcus faecium, Catonella morbi, Clostridium botulinum); this is the most common scenario, and is similar in Gram-negative bacteria, with ula G-ulaA intergenic distances from 250$500 \mathrm{bp}$; (2) ula R and ulaG are co-transcribed along with the rest of structural genes (Lactobacillus casei); in this case, ulaG-ulaCAB intergenic distance shrinks to 5-45 bp, as is expected for genes co-transcribed in the same operon; or (3) ula R coding the DeoR-type regulator is absent, having been replaced by a $b g l \mathrm{G}$ gene encoding a 550-amino-acid residue BglG transcription factor containing two PRD (PTS regulatory domain) domains and an Arc repressor DNA-binding domain (Streptococcus spp.). In the latter case $b g l \mathrm{G}$ is always co-transcribed with ulaG and comes immediately preceding (S. pyogenes) or following it (S. pneumoniae).

We also detected at least four duplicated DNA segments among the multi-species ula regulons examined (Figure 5). The first two cases (P. profundum ulaR and a C. morbi gene coding for an uncharacterized hypothetical protein that is found after ulaF) involve a single gene duplication event which, after divergence, retain about $60 \%$ sequence identity and are co-localized within the operon next to each other on the same strand, and therefore may both be functional in the context of L-ascorbate 6-phosphate degradation. A third case is found in $Y$. intermedia, where the ula regulon lacks a gene for UlaF; instead, two ORFs (yinte0001_31020 and yinte0001_18310) appear to have arisen by gene duplication from an ancestral ula $\mathrm{F}$ gene but these are located in genomic loci far apart from the ula regulon and from one another. Given that the rest of the operon has been conserved, it is plausible that the UlaF-like encoded proteins complement the function lost from the ula regulon. Finally, the most dramatic duplication event was observed in S. termitidis, where the entire regulon (except the gene for the repressor) seems to have undergone a duplication event, the result of which was two reversely transcribed copies. Despite being a Gramnegative bacterium, the ula regulon of $S$. termitidis lacks an ula $\mathrm{R}$ orthologous gene; instead, it contains a BglGencoding gene, similar to that found in Streptococcus spp. Several gene insertions might have occurred since the whole-operon duplication event because the inserted genes were not affected by the duplication; inserted genes include those encoding a sulfatase, a Cof-like hydrolase, and several autotransporters.

In summary, the structure of the ula regulon has been considerably conserved across Gram-negative and Grampositive bacteria despite various species-specific gene rearrangements, fusions, duplications, insertions, and deletions. Although it is not possible to establish beyond doubt that the specific functions of each of the genes in the ula regulon are strictly conserved throughout evolution, the conservation of sequence and operon structure suggests a common functional role for these genes in the catabolism of phosphorylated sugars, mainly L-ascorbate 6-phosphate, via the pentose phosphate pathway.

\section{Gene loss}

In addition to gene duplication, the selective loss of genes in evolving lineages contributes to the diversification of gene inventories [29]. The role of gene loss in the molecular evolution of a protein family within a set 
of closely related genomes can be gauged by comparing the species tree with phylogenies constructed from available protein sequence data. If the existence of an ancestral gene and its vertical transmission to evolving lineages can be stated confidently, then the absence of the gene in a particular descendent lineage can be explained as a gene loss event. In this case, the gene tree will logically lack the particular branch in the species tree where gene loss has occurred. Based on well-documented reports [29], gene loss is generally attributed to negative selection processes that increase the genome's fitness by reducing its size and eliminating dispensable genes. In the case of ulaGL, we first compared our protein tree (Figure 3) with published species trees of Gammaproteobacteria [22,23], which provided evidence for differential loss of ulaGL across several genera. For example, in the enteric Yersinia, some species have lost ulaGL (e.g., Y. pestis) while others (Y. intermedia and $Y$. ruckeri) have retained it. The same pattern of species-specific gene loss is apparent within most genera, like Vibrio and Pasteurella. An extreme case includes both highly specialized bacteria (like the endosymbionts Buchnera and Wigglesworthia) and more distantly related gammaproteobacterial genera (Shewanella, Pseudomonas, Xylella, and Xanthomonas), the genomes of which do not have a detectable ulaGL. This absence may respond to either a lack of ulaGL in the gammaproteobacterial ancestor, or to its presence but subsequent loss due to negative selection pressures.

With the possible exception of the Firmicutes (which are better represented in the UlaGL phylogeny), the presence of ulaGL in a limited set of Gram-positive bacteria from diverse and distantly related phyla such as Actinobacteria, Fusobacteria and Spirochaetes likely reflects gene loss. Within the Firmicutes, the presence of UlaGL in several abundant genera (e.g., Clostridium, Streptococcus, Lactobacillus, and Enterococcus) has many exceptions at the species level that are best interpreted as gene loss events. For example, even though $C$. butyricum, C. botulinum, and C. perfringens all possess an ulaGL gene, most other Clostridium spp. do not, including important human pathogens as C. difficile, C. tetani or $C$. sordellii. It therefore appears that ulaGL has been retained only in particular Gram-positive genomes where it may contribute a fitness advantage.

In all cases analyzed (either Proteobacteria or Grampositive bacteria), loss of ulaGL was always associated with the complete loss of the ula regulon, indicating that the functions conferred by the ula genes are highly coordinated.

\section{Structural homology}

As we have previously discussed [18], the structure of E. coli UlaG strongly resembles RNA-processing enzymes of the MBL superfamily and yet their sequence identity is very low (14-18\%) (Figure 6). Among these, the closest structural homologs found by SSM PDBeFold [30] include $\mathrm{Zn}^{2+}$-dependent RNases that catalyze phosphodiester cleavages in tRNA or mRNA substrates and recognize the three-dimensional structure of their RNA substrates. This group of enzymes includes the premRNA 3'-end-processing endonuclease (PDB ID code 2i7t) [31], tRNA maturase RNase Z (PDB ID code 1y44) [32], and the ElaC homolog of Bacillus anthracis, a putative ribonuclease (PDB ID code 1zkp) (unpublished) (Figure 6 ). The number of aligned residues across all detectable structural homologs was 186 over 13 secondary structure elements, with an overall root mean-square deviation (r.m.s.d.) of $2.1 \AA$ and an overall Q-score of 0.26 . The pattern of conserved secondary structure elements consists of the $\beta$-sheet core (12 $\beta$-strands), except for the two last flanking $\beta$-strands ( $\beta \mathrm{N}$ in $E$. coli UlaG) and the C-terminal helix ( $\beta 14$ in E. coli UlaG). Not unexpectedly, the hexamerization motifs in E. coli UlaG are missing from this conserved core. Further to the global similarity, the RNase Z enzymes appear to show strong similarity to UlaG at the level of the active site configuration and metal coordination sphere, thereby suggesting active-site conservation of the essential features that support hydrolytic activity in both enzyme classes. The similarities observed are remarkable since these RNases come from very broad phylogenetic origins, such as pre-mRNA 3 '-end-processing endonuclease (human) and tRNA maturase RNase Z (bacteria). As noted above, the first iteration of PSI-BLAST with E. coli UlaG as the query had already identified with low scores (24\% coverage, Evalue $0.048,35 \%$ identity over 90 residues) the putative mRNA 3'-end processing factor of the Crenarcheote Pyrobaculum spp. (P. arsenicum and P. islandicum), thus indicating a significant remaining sequence similarity despite enormous divergence.

To find more ancient relatives of UlaG among MBL RNases, we used a structure-guided sequence alignment to construct a profile Hidden Markov (HMM) model [33] to probe the complete non-redundant protein sequence database. Because of the current scarcity of UlaGL protein structures, the number of sequences present in the HMM alignment was necessarily low (6). The search with the HMM pattern retrieved 3929 sequences with a score better than $1 \times 10^{-10}$, which included many sequences already identified by sequence-only searches as well as others from bacterial, archeal, and eukaryotic genomes. Apart from putative lactonases and hydrolases, the search found many sequences for RNase Z, YhfI, AtsA/ElaC, ATP-binding PhnP proteins, lipoate-protein ligase $\mathrm{B}$, aryl- and glycolsulfatases, lipoyl/octanoyl transferases, cAMP phosphodiestarases, tRNA 3' processing enzymes, tRNA endonucleases, and the multidrug 


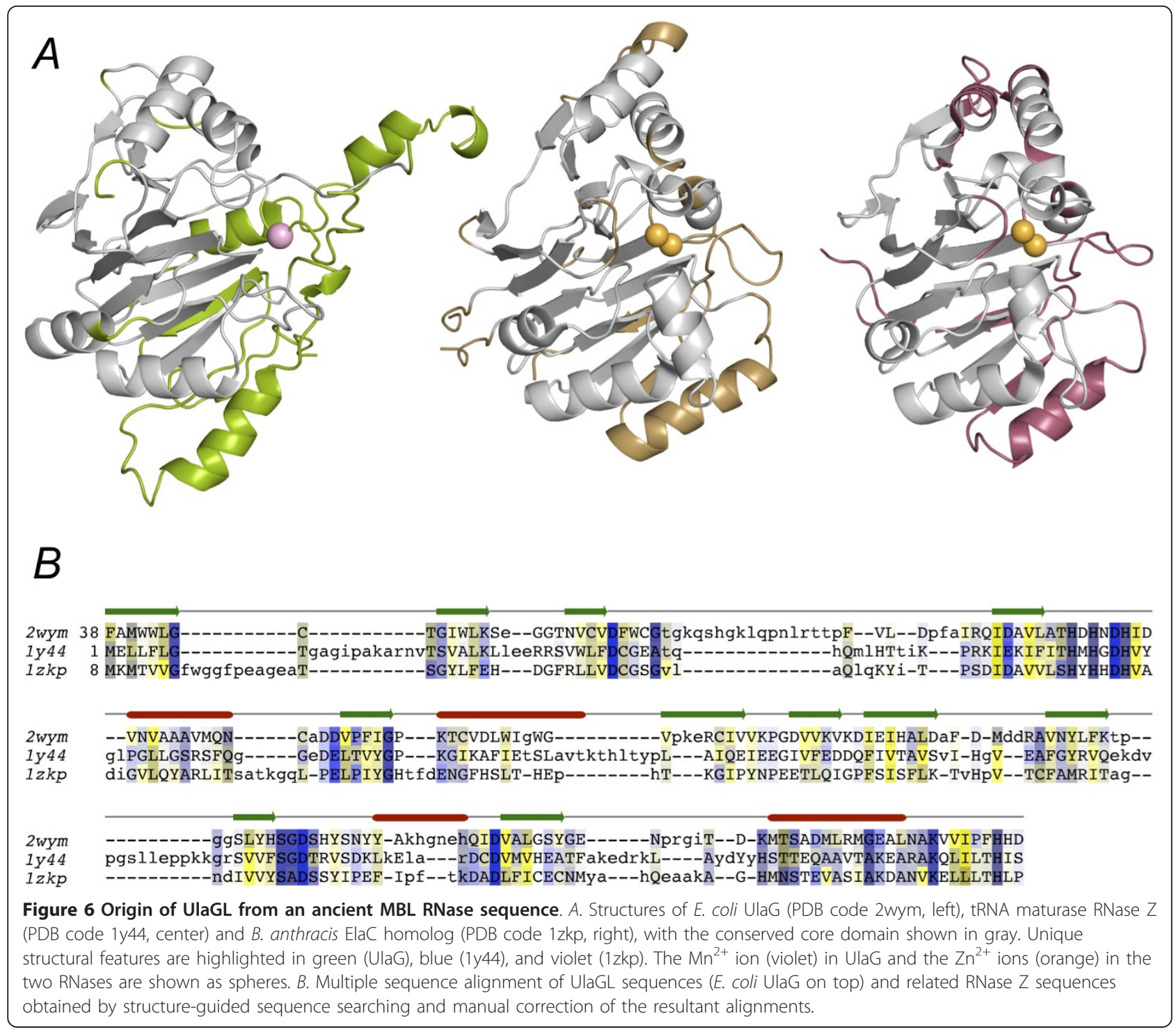

resistance protein RomA. More than $25 \%$ of the sequences were annotated as conserved hypothetical, predicted, or unnamed products, and many of the annotations were not curated (i.e. generated by automatic, database annotation). Despite the poor characterization of most of these sequences, it becomes evident that the descendants of the ancestral sequence from which modern ulaG-like genes originated have a very broad phylogenomic distribution covering Bacteria, Archaea and Eukarya. The HMM searches suggest that the UlaGL sequences in eukaryotic organisms have roles mostly in RNA processing whereas in bacteria, a greater diversity of metabolic and functional roles is appreciable.

Furthermore, the recently determined structure of a MBL from Brucella melitensis subsp. abortus (PDB ID 3md7 and structures thereof) (unpublished) has revealed a monomeric enzyme with an $\mathrm{Mn}^{2+}$-dependent active site similar to UlaG and in contrast to the $\mathrm{Zn}^{2+}$ ligand found in all other RNases. However, this enzyme represents a mixed case since it has only a marginally higher sequence identity with the above RNases (21\%) than with UlaG (15\%). Superposition of the two active sites (not shown) shows that the empty metal site in UlaG is occupied by a second $\mathrm{Mn}^{2+}$ ion in the $B$. melitensis enzyme with a markedly different conformation of the liganded residues. In particular, the two bridging aspartates Asp90 and Asp188 (Asp121 and Asp226 in UlaG) adopt rotamers that bring the carboxylates closer to the $\mathrm{Mn}^{2+}$ (site $\mathrm{Zn} 2$ ) ion by 0.6-1 $\AA$, and two histidine residues in the coordination sphere of the second $\mathrm{Mn}^{2+}$ in the B. melitensis enzyme (site Zn1) are either in a distinct conformation (His88, His119 in UlaG) or have no equivalent in UlaG (His170). The closest residue to His170 in UlaG is Asp184 (2.5 $\AA$ between C $\alpha$ atoms), which is the last amino acid before an extensively 
disordered loop spanning residues 185-204. Only His228 in UlaG has no clear counterpart in the $B$. melitensis enzyme and could in principle play the role of His170; however, the $4.5-\AA$ gap between the corresponding side chains renders this scenario unlikely. The $B$. melitensis structure was solved in the presence of various nucleoside monophosphates (GMP and AMP) that show a similar binding mode, characterized by the nucleotide phosphate buried deep within the metal center of the enzyme and establishing most of the stabilizing interactions, while the ribose moiety and, even less so, the guanine/adenine base, are more loosely bound. Given the chemical and structural similarities of L-ascorbate 6-phosphate with the ribose of GMP and AMP, and the fact that UlaG uses cyclic nucleotide analogs, we hypothesize that L-ascorbate 6-phosphate binds to UlaG in a similar orientation as GMP. This binding mode would position the substrate between the two long loops that are disordered in the UlaG structure, thus raising the possibility of an induced fit mechanism to occur upon substrate binding.

Taken together, structure-based sequence and HMM pattern searches and structure superpositions confirm the evolutionary relationship of the $\mathrm{Mn}^{2+}$-dependent UlaG family and an ancient family of $\mathrm{Zn}^{2+}$-dependent MBLs with RNase activity. Intriguingly, the recently solved structure of a second $\mathrm{Mn}^{2+}$-dependent MBL (from $B$. melitensis) lends further support to the notion that $\mathrm{Mn}^{2+}$ was present in the ancestral RNase or in a wider branch of RNase/metabolic enzymes.

\section{Modeling UlaGL protein structures}

Given the remarkable primary sequence conservation among many UlaGL proteins encoded by the human microbiome, including proteins from recognized human pathogens, we set out to model the hexameric structures of several representative sequences. The chosen set of protein sequences included S. enterica serovar Typhi, C. botulinum, Y. intermedia, S. pyogenes, and A. vaginae. All of these proteins share clear sequence patterns corresponding to the oligomerization motifs (Figures 1 and 2), suggesting that their structures will follow the hexameric architecture revealed by the two crystal structures available for UlaGL proteins [18]. Modeling was carried out with two well-established algorithms (see Methods section) and outcomes were inspected for consistency between the two methods. As controls, modeling of each original crystal structures (PDB ID 2wym and 3bv6) with one another converged to models that were essentially identical to the experimentally determined structure (within r.m.s.d. of 0.3 $\AA$ ). Many sequence changes were conservative and did not generate geometric or electrostatic alterations in the modeled structures. Alterations of this nature might have indicated potential problems with the models. On the contrary, buried surface area (BSA) and other geometric and energetic statistics showed that the modeled structures were capable of forming native interaction surfaces. For example, the average BSA for the largest and second largest interface for the comparative models were, respectively, $4500 \AA^{2}$ and $1250 \AA^{2}$, which compare well with the BSA for the same interfaces from the crystallographic fulllength models (4000 $\AA^{2}$ and $1350 \AA^{2}$ ). Splayed-apart views of the contact surfaces for the modeled structures are shown in Figure 7 and further analyses are described in Additional files 13 and 14.

\section{Prediction of metal ligands}

Prediction of the metal ligand for a particular MBL on the basis of primary sequence and structure alone is errorprone. This is because the same set of protein residues can bind more than one metal in similar or different coordination spheres, but also because the identity of metal ions that can bind into an active site of a particular protein depends, at least in part, on cellular regulatory mechanisms for metal ion homeostasis [34]. To date, four metal ions are known to bind to UlaGL enzymes, including $\mathrm{Mn}^{2}$ ${ }^{+}, \mathrm{Co}^{2+}, \mathrm{Zn}^{2+}$, and $\mathrm{Fe}^{3+}$. For the first three $\mathrm{Mn}^{2+}, \mathrm{Co}^{2+}$, and $\mathrm{Zn}^{2+}$ ) there is strong biochemical and biophysical experimental support [18]. However, the binding of $\mathrm{Fe}^{3+}$ is so far backed only by its presence in an unpublished crystal structure (PDB ID 3bv6), and therefore its biochemical relevance will require a more extensive characterization. In E. coli $\mathrm{UlaG}$, experimental evidence ranks $\mathrm{Mn}^{2+}$ and $\mathrm{Co}^{2+}$ as potential binders, since they display similar binding profile and both facilitate catalysis on L-ascorbate 6phosphate, and singles out $\mathrm{Zn}^{2+}$ as a potential competitive inhibitor of catalytic activity. Interestingly, under the cited experimental conditions E. coli $\mathrm{UlaG}$ does not bind $\mathrm{Fe}^{3+}$ [18]. Collectively, this indicates that the UlaGL family proteins may be able to charge their active site with a broad selection of divalent and trivalent metal ions, thereby suggesting that these perform both carboxyl and phosphoryl ester hydrolysis as well as redox reactions. This versatility in metal selection fits nicely with the expected adaptability of the MBL scaffold fold to catalytically transform a wide range of biologically relevant nutrient molecules.

\section{Discussion}

The recent determination of the X-ray crystal structure of E. coli $\mathrm{UlaG}$, the first enzyme of the anaerobic catabolic pathway of L-ascorbate in Enterobacteria, has brought to light a novel fold type of the MBL superfamily [18]. This sequence and its associated structural features defines a new UlaGL family in the Uniprot database (http://www. uniprot.org) [35]. Much remains unknown about its phylogenetic origin, its evolutionary relatedness to RNA processing enzymes, and the potential plasticity of the structural elements that configure UlaG. In order to increase our understanding of the UlaG family we sought to study its 


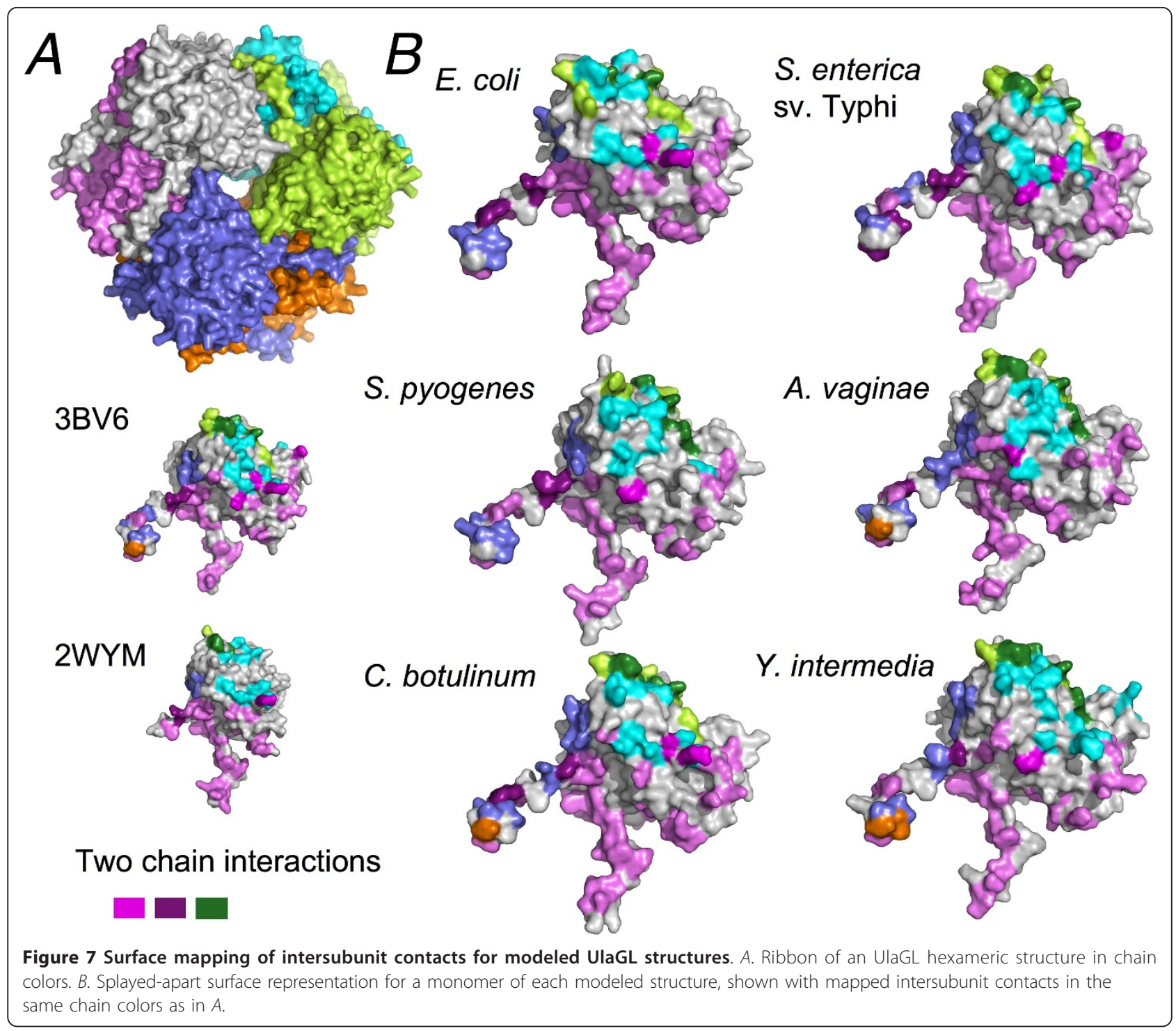

phylogenetics and the consequences of the colocalization of the corresponding gene with genes coding proteins associated with the ula regulon. Here, we have retrieved and analyzed a set of near 200 bacterial and archeal sequences with detectable homology to UlaG and that we have denominated the UlaGL family.

A first set of closely homologous sequences prevails in related Gram-negative bacteria from the Gammaproteobacteria that include species from the orders Enterobacteriales, Vibrionales and Pasteurellales. Many, if not all, of these sequences come from bacteria from the mammalian microbiome and also from human, animal, and plant pathogens. Given their relatively high amino acid sequence identity ( $>60 \%$ ) and the likely presence of a shared genomic context homologous to genes in the ula regulon, the UlaG homologs constitute plausible orthologous sequences. Even relatively distant bacteria such as
$V$. cholerae, an obligate anaerobe and human pathogen, conserves the ula regulon structure and has sequence identity to E. coli UlaG above 65\% (Figure 2, Additional file 3), in addition to sharing the same structural scaffold with an r.m.s.d. of $1.5 \AA$ for nearly the entire length of the two proteins. This strong pattern of conservation of the sequence and structure of both the structural genes and the regulatory sequences of the ula regulon across Gammaproteobacteria is in agreement with the evolution of Enterobacteria, and indicates that these sequences are of ancient origin [36-38] and under strong purifying (negative) selection [3]. Assuming vertical transmission of ulaGL among the Gammaproteobacteria, the lack of ula GL along certain genera and species (e.g. Y. pestis and C. tetani lack the ula regulon) indicates a selective loss of the ulaGL gene present in the ancestor to those lineages, or absence in the ancestor followed by acquisition from a 
distant source in several lineages. Given that HGT between closely related species is rare [29] and the congruence of the gene tree with the species tree for gammaproteobacterial genomes, the first scenario, involving gene loss of an ancestral gene, appears more likely to have played a significant role in shaping the phylogenomics distribution of the UlaGL family.

A second set of homologous UlaGL sequences are found in Gram-positive bacteria (mostly Firmicutes and Actinobacteria), again mainly among symbiotic and pathogenic mammalian-associated bacteria. A syntenic association between ulaGL genes and an adjacent ulaR-like gene is also strongly conserved in Gram-positive bacterial genomes, although in Streptococcus spp. the ulaR-encoding DeoR repressor appears to have been substituted by a gene coding a more complex BglG-family transcription factor, which has two PRD (PTS regulatory domains) and an Arc DNA-binding domain. While the defining features of the ula regulon are conserved among Gram-negative and Gram-positive bacteria, there are numerous speciesspecific gene rearrangements and insertions, and even whole operon duplications, which might play adaptive roles in the particular lifestyle and metabolic requirements of each species. Thus, the plasticity of the ula regulon structure is in agreement with previous reports that suggest that gene sequences are generally more conserved than the gene organization within an operon, with the possible exception of genes encoding physically associated polypeptide chains, which tend to have a higher propensity to remain clustered [22].

The conservation of genomic context about the putative ula GL regulons/operons within closely related species indicates a similar functionality in carbohydrate/vitamin metabolism. Indeed, the presence of sequences of prophage origin flanking the ula regulon in several enterobacterial species and the flanking transposase/integrase genes found in Yersinia spp. are a strong indication of the coherent function as well as the potential for the lateral transfer of the entire regulon, and are consistent with the HGT events from an enterobacterial donor to Gram-positive recipients apparent from the reconstructed molecular evolution of ulaGL (Figure 3). When larger evolutionary distances are considered, the genomic context becomes far less conserved. For example, for more distantly related Gram-negative species such as Escherichia spp. and Yersinia spp. the genome location of the ula regulons is unrelated.

Most interestingly, the combination of structural and sequence homology searches help delineate a complete phylogenetic tree of UlaG-related sequences with a broader scope than any individual approach might achieve. A first feature of such a tree is a core of bacterial sequences that share a closely related functionality in L-ascorbate 6-phosphate (or a similar metabolite) metabolism and which span Gram-negative and Grampositive bacteria with diverse lifestyles (commensal, symbiotic, pathogenic, or free living). Although true orthology is a plausible scenario between UlaGL sequences with $>55 \%$ sequence identity with the $E$. coli reference sequence, at this stage we cannot exclude that in some organisms the ula operon was dedicated to the catabolism of other, related metabolites, such as a different hexose phosphate. Subtle changes in the active site might change the identity of the bound metal or the catalytic side chains, thereby modifying substrate specificity and/or reaction selectivity. Furthermore, the environment of the microorganism and its metal homeostasis [34] constrains the possible substrates, in addition to creating selection pressures in favor of particular metabolic outcomes. Therefore, the question of the specific function, among the relatively narrow set of choices compatible with the phylogenetic analysis, of the ula operon in each organism should be addressed in a species-specific fashion.

The combination of sequence searches with structural information has allowed the restoration of the distant evolutionary history of UlaGL proteins, whereby the most prominent feature is the relatedness of modern metabolic UlaGL enzymes with the widespread RNase Z essential enzyme. This relation strongly suggests that the forerunner to the modern UlaGL sequences was an RNase that was present in the last common ancestor of Eubacteria, Archea, and Eukarya. Key differences between these two protein families are the acquisition by UlaGL of $\mathrm{N}$ - and/or $\mathrm{C}$-terminal sequences governing their quaternary architectures and the presence of multiple insertions/deletions in the loops that extend over the active site. The abstraction of the conserved sequence-structure features between UlaGL and RNase Z permits the reconstruction of even more distant (diverged) phylogenetic relationships of UlaGL with $\mathrm{Zn}^{2+}$-dependent hydrolases and oxidorreductases. The latter enzymes have previously been noted, on the basis of strict structure conservation, to form an independent lineage loosely termed class B3 $\beta$-lactamases and distinct from class $B 1+B 2 \beta$-lactamases [14]. Although structure-based profiles and sequence alignments were required to reliably establish the evolutionary relatedness between UlaGL and RNase Z, it is interesting to consider that the accumulation of high-quality sequencing data for many different species made it possible to retrieve at least one RNase $\mathrm{Z}$ sequence on iteration 1 (and later iterations) from sequence-only searches of E. coli UlaG using PSIBLAST. This sequence is from the Crenarcheota Pyrobaculum spp., with E-score of $1 \times 10^{-4}, 24 \%$ sequence identity and 40\% sequence similarity (over 201 amino-acid residues). Another putative homologous sequence that appears in PSI-BLAST searches but whose reliability has not been assessed owing to a lack of a crystal structure is 
the membrane protein RomA, which participates in multidrug efflux. Interestingly, RomA from diverse pathogenic bacteria appears repeatedly in PSI-BLAST searches with scores that are marginally better to those of Pyrobaculum spp. RNase Z (E-score $7 \times 10^{-4}, 25 \%$ sequence identity, $41 \%$ sequence similarity, over 269 amino-acid residues). Finally, direct structure comparison will be required to establish that UlaGL and RomA transporters share a common ancestor and to rule out other possible scenarios for the sequence similarity observed.

Some structural features of UlaG stand in stark contrast with the overall fold plan of MBL RNA-processing enzymes, including the oligomerization motifs and the presence and location of putative substrate-binding elements. This distant homology detected only at the structural level allows us to put forward a hypothesis whereby the ancient fold type of MBLs, possibly devoted to an essential function in relation to RNA processing, has been duplicated and modified to accommodate new substrate specificities and new reactivities. Indeed, UlaG still retains measurable phosphodiesterase activity toward cyclic nucleotides as a side reaction [18].

Our phylogenetic analysis of the UlaGL family has allowed the definition of the structural and sequence features constituting the UlaGL fold and that differentiate it from other related MBLs. Since these features are derived in part from the available crystal structures and in part from systematic sequence searches, they have predictive power for comparative modeling for sequences from unknown structure. Indeed, most UlaGL close homologs can be confidently modeled (Figure 7). Our modeling exercise has confirmed that the distinctive hexameric complex is well conserved. The buried surface areas, surface complementary, and number of interface interactions are no less indicative of a strong interaction than for the two reference structures. Further details on the modeled sequences and evidence that the structures could exist as hexameric complexes are given in Additional files 13 and 14 . No attempt was made to model sequences that did not have identities above $50 \%$ and for which the similarity did not extend along the complete length of the protein sequence.

The structure-guided phylogenetic analysis that we have performed shows that the diversity of sequences related evolutionarily to UlaG is much greater than previously anticipated. UlaGLs are present in several bacterial lineages, from mammalian symbionts to free-living marine bacteria, and are found even among archeal and eukaryotic sequences. By combining available crystal structures with molecular evolution methods we have provided solid support to the idea that the modern UlaGL family of carbohydrate and vitamin metabolism originated from an ancient RNA processing enzyme by the addition of $\mathrm{N}$ and $\mathrm{C}$-terminal oligomerization motifs and the specialization of its active site. The spread of UlaGL (along the ula regulon) among symbiotic and pathogenic bacteria by HGT, which is supported by our Bayesian and maximum likelihood tree reconstructions, suggests that UlaGL may have conferred a growth advantage under some environmental and community pressures. Finally, the analysis presented here, combined with future ones gathered from all sequence and structure data that will be generated, will afford a better understanding of the UlaGL family and, by extension, of other subfamilies of the wide MBL trunk of sequence space.

\section{Conclusions}

The emergence of new functions from pre-existing folds by gene duplication and divergence of the new copy represents an enormous source of genetic variation in prokaryotic genomes, which is further expanded by HGT and genomic rearrangement events. The gene encoding UlaG in commensal and symbiotic enterobacteria of the human gastrointestinal tract is an example of the result of an ancient gene duplication event from a gene which may have had an essential RNA metabolizing function in the last common ancestor of Bacteria, Archaea, and Eukarya. Our phylogenetic analyses, based both on sequence-only and structure-guided multiple sequence alignments, provide solid support for this hypothesis, and reveal a greater phylogenetic distribution for genes encoding UlaGL than previously anticipated, including the possibility of multiple independent gene duplications along bacterial and archeal lineages, as well as of lineage-specific gene loss. On the basis of these analyses, we propose that the ancestral RNase-encoding gene adopted its new metabolic role in the context of L-ascorbate metabolism by grafting both $\mathrm{N}$ and C-terminal motifs onto the RNase scaffold while extensively modifying the active-site loops responsible for binding the metal and substrate(s) and exerting the catalytic function(s). This scenario, i.e. the creation of new functions by modification of the extremes and critical functional loops in the core of a pre-existing MBL domain, as well as by selection of a more suitable metal ion (an aspect frequently overlooked in MBL enzymes in favor of an assumed $\mathrm{Zn}^{2+}$ metal center), might provide clues as to the parts of an MBL protein sequence that can accommodate most variation and therefore represent important determinants of, for example, the generation of new antibiotic resistance mechanisms.

\section{Methods}

\section{Generation of the UlaGL sequence data sets}

In order to restrict the initial phylogenetic analysis to the set of core UlaG orthologs, we searched the NCBI nr database with BLASTP [39] applying an E-value $<1 \times 10^{-10}$ and a match length $>75 \%$ of the query length (265 amino acids). Of the 220 sequences found to fulfill the E-value 
criterion, $91 \%$ or 201 sequences had an aligned length greater than 265 residues. This last criterion proved crucial to prune sequences that retained homology to the core MBL domain while having lost several UlaG characteristic features, such as the 20 -amino-acid residue $\mathrm{N}$ terminal and the $\mathrm{C}$-terminal oligomerization motifs. The 201 single copy genes that met these homology criteria were further trimmed on the basis of sequence identity (sequences with $>50 \%$ sequence identity were retained) and pruned from duplicated sequences; the resultant group, containing 175 sequences, was used for phylogenetic reconstruction. Concatenated sequences of these 175 genes were aligned using ClustalX v. 10.0.2 [40] or Muscle v. 3.6 [41] and Gblocks v. 0.91b [42] was applied to edit the alignment to remove gaps and poorly aligned regions. The final alignment contained 333 aligned amino acid characters of the original 415 (80\%).

We then sought to find more distantly related UlaGL sequences by searching the NCBI protein and nucleotide sequence databases for fully sequenced genomes (December 2008) using either PSI-BLAST (BLASTP v. 2.2.24+, non-redundant protein databases, 1000 maximum target sequences, profile-inclusion threshold of expected Evalue 0.005 , four iterations) or tBLASTn (translated nucleotide databases) from the NCBI Blast2 interface (http://blast.ncbi.nlm.nih.gov/Blast.cgi) [39]. After the initial sequence collection, the full NCBI database was probed with individual UlaG homologous sequences to enhance the chances of Finding sequences related to particularly divergent MBLs and to better define the range of organisms containing these proteins. Sequences were aligned with ClustalX or Muscle with default alignment parameters and unnecessary gaps and poorly aligned regions were pruned with Gblocks. The subset of archeal UlaGL homologs were selected from the original multispecies alignment, re-aligned to emphasize archeal-specific sequence features, and prune with Gblocks for phylogenetic purposes. The archeal UlaGL alignment had 264 aligned amino acid characters from 17 taxa.

To retrieve even more distant UlaGL sequences, we first constructed a structure-based sequence alignment using the superimposed homologous structures identified with PDBeFold [30] (see below) and used this alignment, with 5 sequences and 232 aligned amino acid residues, as seed for the calculation with HMMER v. 3.0 (http://hmmer.org/) of a profile hidden Markov model (HMM) [33]. This HMM profile was then queried against the NCBI nr database with default parameters to retrieve sequences with significant similarity to the profile HMM. For a representative subset of 30 RNase Z sequences identified by HMMER with high E-values (< $1^{-10}$ ) we built a pruned multiple sequence alignment as described above (Figure 6).
A complete listing of bacterial sequences, taxonomy, accession numbers and their sequence identities with respect to E. coli UlaGL is provided as Additional file 3. The complete sequence sets and corresponding alignments are provided as Additional files 4, 5 and 6, and 9, 10 and 11.

\section{Phylogenetic tree generation}

Maximum likelihood (ML) and Bayesian Inference (BI) phylogenetic analysis were carried out with RAxML v. 7.0.4. [43] and MrBayes v. 3.1.2 [44], respectively, using the concatenated multi-species alignment files for the complete UlaGL bacterial orthologous sequences (Figure 3 ), the archeal homologous sequences (Figure 5), and the distant MBL RNase $\mathrm{Z}$ alignment (Figure 8). RAxML was run with the PROTCATWAG model, with 100 bootstrap replicates. Bayesian consensus trees were generated with MrBayes using amino acid substitution rates and state frequencies fixed to the WAG parameters [45]. A uniform $(0.0,200.0)$ prior was assumed for the shape parameter of the gamma distribution of substitution rates [46], an unconstrained exponential prior with rate 10.0 for branch lengths, and all labeled topologies were a priori equally probable. Four independent MCMC analyses were run, each with one cold chain and three heated chains, with the incremental heating schema implemented in MrBayes $(\lambda=0.2)$. Convergence was assumed after the topology of the samples from the two cold chains had reached an average standard deviation of split frequencies (SDSF) of less than 0.01 (after $5,032,000$ generations). The resulting trees were drawn with FigTree (http://tree.bio.ed.ac.uk/software/figtree/). No significant changes in the topology of the trees were observed when comparing the RaxML and MrBayes results, although differences in statistically unsupported regions of the trees were common.

In addition to the complete bacterial UlaGL tree, a tree containing a set of gammaproteobacterial species was generated with Tree-Puzzle v. 5.2 [47] using established methods $[22,23]$. The congruence between the UlaGL tree and the species tree was assessed by the Shimodaira-Hasegawa ( $\mathrm{SH}$ ) [48] test as implemented in the same software package.

All phylogenetic trees are provided as Additional files 7, 8 and 12 .

\section{Genomic context}

Genomic context was retrieved from the Entrez database [49], where records were available, or via the CDS records with the NCBI genome entry. Transcription orientation was consulted with EnsemblBacteria (http:// bacteria.ensembl.org/) or the Genome Reviews tool (http://www.genomereviews.ebi.ac.uk/). 


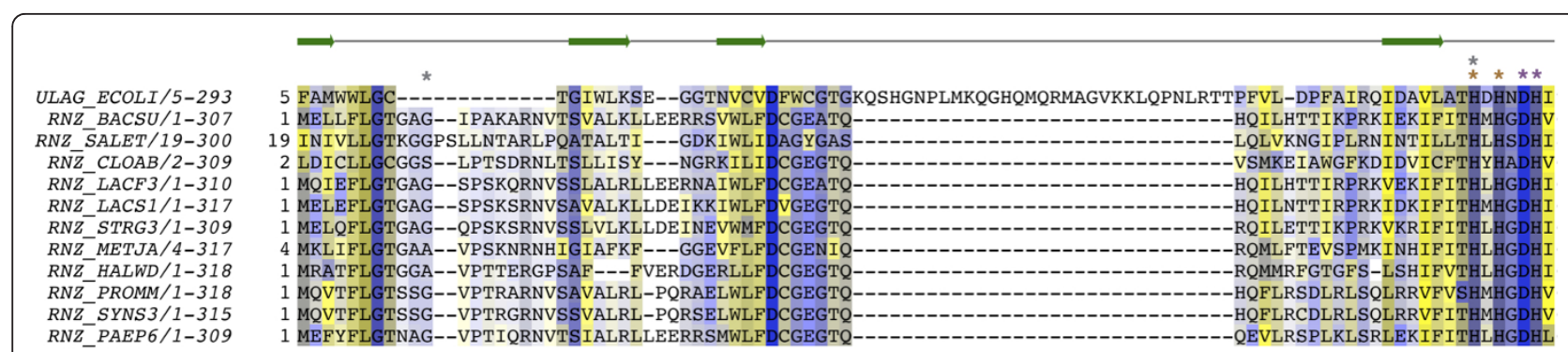

$U L A G \_E C O L I / 5-293$ $R N Z_{B A C S U / 1-307}$ RNZ_SALET/19-300 $R N \bar{Z}$ C CLOAB/2-309 RNZ LACF 3/1-310 $R N Z$ LACS 1/1-317 RNZ RNZ_STRG $3 / 1-309$ RNZ_METJA $/ 4-317$
RNZ_HALWD/1-318 $R N Z$ HALWD/1-318
$R N Z \_P R O M M / 1-318$ RNZ_PROMM $/ 1-318$ RNZ_PAEP6/1-309

\begin{tabular}{|c|c|}
\hline & * \\
\hline L-D-VNVAAAVMQN-CAD--DVPFI & $-----V P K E R C I V V K P-$ \\
\hline 0 YGLPGLLGSRSFQGGED--ELTVYG & PKGIKAFIETSLAVTKTHLTYPL---AIQEIEE--------GIVFEDDQFIVTAVSVIH------------------G \\
\hline 7 LDYPSLLMNAWASGLKDH-TIQVYG & 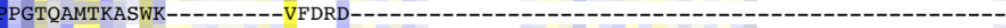 \\
\hline 68 MGLTGLLLTIANSGRID--PLTIIG & PEGLREVVKG-LTVVAPFFPYEI---ELIELDSKCSDNFLDKVFKIEDVEIFALPVDH- . \\
\hline 70 FGLPGFLSSRSFQGGDE--PLTIYG & PKGIKDFVQTALKVSESRLSYPL---KFVELTG----D--GEVFKDQTFTVTARRLDH-------------------K \\
\hline 70 FGLPGFLSSRSFQGGEKMGPLTIYG & 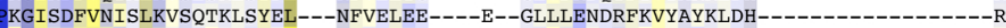 \\
\hline 70 FGLPGFLASRAFQANEEQTDLDVYG & 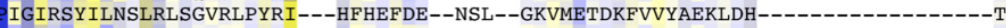 \\
\hline 70 LGIPGLLQSMGFFGREK--ELKIFG & PEGTKEI IENSLKLGTHYIEFPI---KVYEIYT--KEP--ITIYKEENYEIIAYPTEH---------- \\
\hline 66 LGIPGLIQTLDFNDRTD--ALAIHG & PFGSKPHLRHLVETAGYRPGYPI---NIREIRP-----G--SVAVDTEEYIIRSFETSH-- \\
\hline 69 FGLPGLLASLGLSGNSN--GVDLYG & PDPLESYLQGVLRNSSTRIGYPL---KVHRVRDAAEQN--LIVFEDKDILVRCTPLTH-------- \\
\hline 9 FGLPGLLASLGLGSTSN--GVDLYG & PDLDAYLQGVLRTSSTRIGYPL---AIHRVREAAEQN--TVVFEDDDLIVTAAPLNH- \\
\hline & \\
\hline
\end{tabular}

ULAG_ECOLI/5-293 171 GMMDDRAVNYLFKTP

*

RNZ BACSU/1-307 142 -V--EAFGYRVOEKDVPGSLKADVLKEMNIPPGPVYO-KIKKGETVTLEDGRI INGNDFLEP-PKKGRSVVFSGDTRVSDKLKELA---RDCDVLVHEATFA

RNZ $\bar{S} A L E T / 19-300129$-IT-LRMEEEGKPDPRNLVKATDIG--OGVIYKDELVTISALKVPHSPFPDGEAFAYRFDTOGKRIVFSGDTSWFPPLATFAO-GADILVHEAVHVPSVAKL $R N \bar{Z} C L O A B / 2-309146$-I--ECLSYSVR-VNRKRKFDVNKAKANEVPLK-IWN-ELORGKEITYENKLYV-PDMVLGE-SRKGIKITYCTDTRPVDSLHKFA---YKSDLFVCEGMYG RNZ LACF3/1-310 143 -I--ASFGYRVEEAAHPGELMVEKVROAGIPSGPLYG-OLKRGEVVTLPDGRTVDGHDFIGA-POPGRIVAILGDTRVTDNAVKLA---KGADVLVHEATFA RNZ LACS1/1-317 145-I--ECWGYRIEEKDYPGELOVEKLREAKVPSGPIYG-RLKAGEVVTLEDGRTIDGKDFIGK-AKKGRIVTILGDTROTPNLKLLA---KDADVLVHESTFG RNZ_STRG3/1-309 147 -I--FCIGYRVMQKDLEGTLDAEALKAAGVPFGPLFG-KIKSGQDVILEDGTKIIAKDYISA-PKKGKIITILGDTRKTDASVRLG---LGADILVHESTYG RNZ_METJA/4-317 145 -I--PSYAYIFKEIKKP-RLDIEKAKKLGVKIGPDLK-KLKNGEAVKNIYGEI IKPEYVLLP-PKKGFCLAYSGDTLPLEDFGKYL---KELGCDVLIHEAT $R N Z_{-} H A L W D / 1-318139$ GV--TSIGYALIESDRKGRFNRDRAESLGVPVGPKFG-RLHDGESVKLADGSIVHPEQVVGD-SRPGRRVVYTGDTRPTETTVEAA---NNADLLVHDATFA

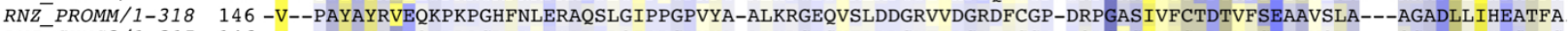
RNZ_SYNS3/1-315 146 -V--PAYAYRAEQKPRAGRFDIDKARELQIPPGPVYA-ALKRGESVTLDDGRTIDGRTLCGP-EQPGVSVVYCTDTVFCEAAVQLA---QGADLLIHESTFS RNZ PAEP6/1-309 142 -I--DSYGYRVVEMDKPGKLNQELLERYGIKPGPVYG-KLKRGESVEVEGGVILHPQDVLGS-PKKGRIITILGDTRPCPNTVVLA---QDATVVVHEATFL

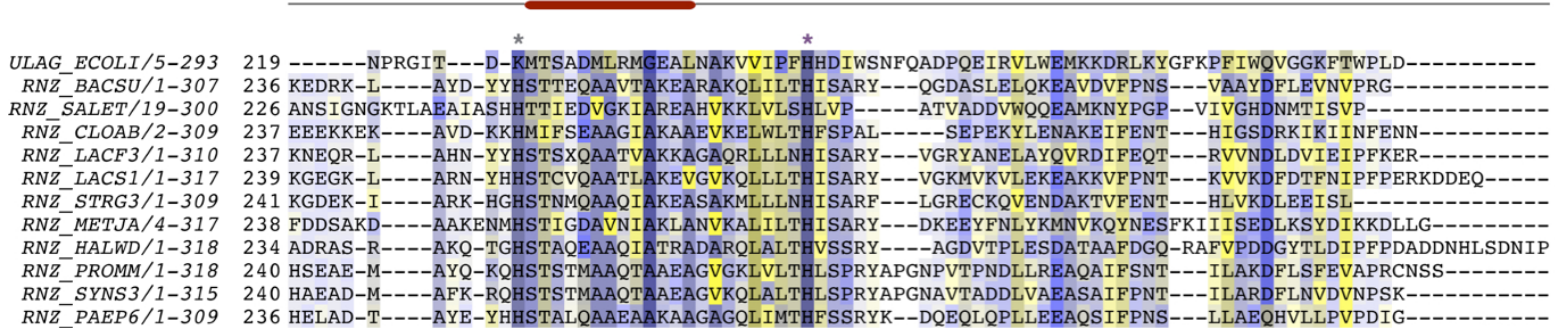

Figure 8 Sequence alignment of UlaGL/RNase Z homologs. Multiple sequence alignment of E. coli UlaG and homologous RNase $Z$ sequences from selected Bacteria and Archea, with secondary structure overlaid above. Residues in contact with $\mathrm{Mn}^{2+}$ in E. coli UlaG are indicated with gray asterisks, and key histidine and aspartate residues in MBL conserved motifs I-V are marked with orange and purple asterisks, respectively.

\section{Structural comparisons and modeling}

Structural homologs of UlaG were found with PDBeFold [30] using the crystal structures of apo- and $\mathrm{Mn}^{2+}$-loaded E. coli UlaG (PDB ID 2 wyl and 2 wym, respectively) [18] with default cut-off parameters (70\% identity of secondary structure in the target and the query) as search probes. Modeling of homologous hexameric UlaGL structures were performed with MODELLER-9v8 [50] and Rosetta v. $3.2[51,52]$ using customized python scripts and pair-wise sequence alignments extracted from the multiple sequence alignments used for phylogenetic analyses (see above). In MODELLER-9v8, 100 models were generated using the standard 'automodel' with additional symmetry restraints to keep similar conformations for each chain of the hexamer. The models were assessed using Z-DOPE, a normalized atomic distance-dependent statistical potential based on known protein structures. The Z-DOPE scores ranged from -1.11 to -1.62 , where a score less than -1 indicates a 'reliable' model (i.e., $80 \%$ of its $\mathrm{C} \alpha$ atoms are within $3.5 \AA$ of their correct positions). Hexameric models with the best Z-DOPE scores were subjected to loop refinement of the residues involved in 
the oligomerization motifs (Figure 1). For each loop, 50 models were generated using the 'loop' routine of MODELLER (with additional symmetry restraints, as above). The final loop conformations were then selected based on their Z-DOPE scores. Finally, the best models were subjected to side chain refinement using SCWRL4 [53]. Although some post side chain refinement models had worse Z-DOPE scores than unrefined models, they exhibited better stereochemistry (Additional file 13) and are therefore likely to be more accurate than the unrefined models. The Z-DOPE scores for the final refined hexameric models ranged from -1.20 to -1.68 . Not surprisingly, the control hexameric crystal structures (PDB codes 2 wym and 3 bv6) received the best scores $(-2.10$ and -1.84 , respectively). Stereochemistry was assessed using MolProbity [54]. In addition, for each hexamer model, the interfaces were evaluated with ModTie, a residue contact statistical potential derived from binary domain interfaces in known structures (Additional file 13) [55]. Interface $Z$ scores that are less than -2 indicate a 'possible' surface. The interfaces of all hexamers scored lower than -2, and the scores were not appreciably correlated with the percent identity to the template structures. The analysis of surface area and shape complementarity (Additional file 14), as well as all figures showing structures and superpositions, were carried out with PISA [56] (http://www.ebi.ac.uk/msd-srv/prot_int/pistart.html) and PyMOL (http://www.pymol.org/).

\section{Additional material}

Additional file 1: Figure S1. Genetic organization and transcriptional direction of the utilization of L-ascorbate (ula) regulon in Escherichia coli and encoded structural genes.

Additional file 2: Figure S2. Catabolism of L-ascorbate 6-phosphate in enterobacteria and established catalytic activity of E. coli UlaG.

Additional file 3: Table S1. Sequences included in the phylogenetic analyses.

Additional file 4: Bacterial UlaGL sequences. FASTA file containing the complete set of bacterial UlaGL sequences.

Additional file 5: Filtered multiple sequence alignment of bacterial UlaGL sequences. Multiple sequence alignment of bacterial UlaGL sequences in FASTA format prior to Gblocks filtering.

Additional file 6: Unfiltered multiple sequence alignment of bacterial UlaGL sequences. Multiple sequence alignment of bacterial UlaGL sequences in FASTA format after Gblocks filtering, used for phylogenetic reconstruction.

Additional file 7: Phylogenetic tree of bacterial UlaGL. Majority rule consensus tree of bacterial UlaGL sequences calculated with MrBayes and RAxML (used to prepare Figure 3). Nodes are labeled with BIPP (Bayesian inference posterior probability) and MLBP (maximum-likelihood bootstrap percentage).

Additional file 8: Gammaproteobacterial species tree used for comparison with UlaGL tree. Phylogenetic tree constructed from a set of representative Gammaproteobacteria species that encode UlaGL, used for the evaluation of potential HGT events between related gammaproteobacterial species. Internal nodes are labeled with maximum-likelihood branch length support (MLBL). Tree was calculated with Tree-Puzzle v. 5.2

Additional file 9: Archeal UlaGL sequences. FASTA file containing the set of archeal UlaGL sequences.

Additional file 10: Unfiltered multiple sequence alignment of archeal UlaGL sequences. Multiple sequence alignment of archeal UlaGL sequences in FASTA format prior to Gblocks filtering.

Additional file 11: Filtered multiple sequence alignment of archeal UlaGL sequences. Multiple sequence alignment of archeal UlaGL sequences in FASTA format after Gblocks filtering, used for phylogenetic reconstruction.

Additional file 12: Phylogenetic tree of archeal UlaGL. Majority rule consensus tree of archeal UlaGL sequences (using two eubacterial sequences as outgroups) calculated with MrBayes and RAxML (used to prepare Figure 5). Nodes are labeled with BIPP (Bayesian inference posterior probability) and MLBP (maximum-likelihood bootstrap percentage).

Additional file 13: Table S2. Homology modeling, interfaces, and geometry validation of UlaGL homology models generated with MODELLER-9v8.

Additional file 14: Table S3. Analysis of buried contact area by PISA of reference structures and homology models generated with MODELLER-9V8.

\section{List of abbreviations}

(BSA): buried surface area; (GIT): gastrointestinal tract; (HGT): horizontal gene transfer; (HMM): Hidden Markov model; (MBL): Metallo-beta-lactamase; (r.m.s. d.): root mean-square deviation; (TIM): Triose phosphate isomerase; (ula): utilization of L-ascorbate; (UlaGL): UlaG-like.

\section{Acknowledgements}

This work was supported by the Spanish Ministry of Science and Innovation (MICINN) (grants PET2008_0101, BIO2009-11184 and BFU2010-22260-C02-02 to MCV, grant BFU2010-22260-C02-01 to LB, and grants BFU2008-02372/BMC and CSD2006-00023 to MC), the Spanish National Research Council (CSIC) (grant PIE-2008-201056 to MCV), and the Generalitat de Catalunya (grant 2009SGR-1309 to MC). FJF and MLE were supported by the MICINN grant PET2008-0101 and a fellowship (ME-517217) from the Spanish Ministry of Education, respectively. MLE acknowledges the support of the Ph.D. program in Biochemistry, Molecular Biology and Biomedicine of the Universidad Complutense de Madrid (UCM). The authors thank the Galicia

Supercomputing Center (CESGA) for the provision of computational support.

\section{Author details}

${ }^{1}$ Structural and Quantitative Biology Department, Centro de Investigaciones Biológicas (CIB-CSIC), Ramiro de Maeztu 9, 28040 Madrid, Spain. ${ }^{2}$ Genome Damage and Stability Centre, University of Sussex, Falmer, Brighton, BN1 9RQ, UK. ${ }^{3}$ Departament de Bioquímica i Biologia Molecular, Institut de Biomedicina de la Universitat de Barcelona (IBUB), Facultat de Farmàcia, Universitat de Barcelona, 08028 Barcelona, Spain. ${ }^{4}$ Institut de Biologia Molecular de Barcelona (IBMB-CSIC), Barcelona Science Park, Baldiri Reixac 10, 08028 Barcelona, Spain. Institute for Research in Biomedicine (IRB Barcelona), Barcelona Science Park, Baldiri Reixac 10, 08028 Barcelona, Spain.

\section{Authors' contributions}

FJF performed most structural and phylogenetic analyses and edited the manuscript. FG and MLE participated in the structural analysis. JA, LB, MC and JB edited the manuscript. MCV conceived and designed the overall study, supervised all analyses and wrote the manuscript. All authors read and approved the final manuscript.

Received: 21 June 2011 Accepted: 26 September 2011 Published: 26 September 2011

\section{References}

1. Lynch M, Conery JS: The evolutionary fate and consequences of duplicate genes. Science 2000, 290(5494):1151-1155. 
2. Ohno S: Evolution by Gene Duplication. Heidelberg, Germany: SpringerVerlag; 1970.

3. Price MN, Dehal PS, Arkin AP: Horizontal gene transfer and the evolution of transcriptional regulation in Escherichia coli. Genome Biol 2008, 9(1):R4.

4. Juhas M, van der Meer JR, Gaillard M, Harding RM, Hood DW, Crook DW: Genomic islands: tools of bacterial horizontal gene transfer and evolution. FEMS Microbiol Rev 2009, 33(2):376-393.

5. Chothia C, Gough J, Vogel C, Teichmann SA: Evolution of the protein repertoire. Science 2003, 300(5626):1701-1703.

6. Hall $B G, X u L$ : Nucleotide sequence, function, activation, and evolution of the cryptic asc operon of Escherichia coli K12. Mol Biol Evol 1992, 9(4):688-706.

7. Olson MV: When less is more: gene loss as an engine of evolutionary change. Am J Hum Genet 1999, 64(1):18-23.

8. Moran NA: Microbial minimalism: genome reduction in bacterial pathogens. Cell 2002, 108(5):583-586.

9. Marais GA, Calteau A, Tenaillon O: Mutation rate and genome reduction in endosymbiotic and free-living bacteria. Genetica 2008, 134(2):205-210.

10. Giovannoni SJ, Tripp HJ, Givan S, Podar M, Vergin KL, Baptista D, Bibbs L, Eads J, Richardson TH, Noordewier M, et al: Genome streamlining in a cosmopolitan oceanic bacterium. Science 2005, 309(5738):1242-1245.

11. Xu J, Mahowald MA, Ley RE, Lozupone CA, Hamady M, Martens EC, Henrissat B, Coutinho PM, Minx P, Latreille P, et al: Evolution of symbiotic bacteria in the distal human intestine. PLoS Biol 2007, 5(7):e156.

12. Schmidt MA: LEEways: tales of EPEC, ATEC and EHEC. Cell Microbiol 2010, 12(11):1544-1552.

13. Jackson RW, Johnson L, Clarke SR, Arnold DL: Bacterial pathogen evolution: breaking news. Trends in genetics: TIG 2011, 27(1):32-40.

14. Bebrone C: Metallo-beta-lactamases (classification, activity, genetic organization, structure, zinc coordination) and their superfamily. Biochem Pharmacol 2007, 74(12):1686-1701.

15. Ma BG, Chen L, Ji HF, Chen ZH, Yang FR, Wang L, Qu G, Jiang YY, Ji C, Zhang HY: Characters of very ancient proteins. Biochem Biophys Res Commun 2008, 366(3):607-611.

16. Vega MC, Lorentzen E, Linden A, Wilmanns M: Evolutionary markers in the (beta/alpha)8-barrel fold. Curr Opin Chem Biol 2003, 7(6):694-701.

17. Ranea JA, Sillero A, Thornton JM, Orengo CA: Protein superfamily evolution and the last universal common ancestor (LUCA). J Mol Evol 2006, 63(4):513-525.

18. Garces F, Fernandez FJ, Montella C, Penya-Soler E, Prohens R, Aguilar J, Baldoma L, Coll M, Badia J, Vega MC: Molecular architecture of the Mn2 +-dependent lactonase UlaG reveals an RNase-like metallo-betalactamase fold and a novel quaternary structure. J Mol Biol 2010, 398(5):715-729.

19. Garces F, Fernandez FJ, Perez-Luque R, Aguilar J, Baldoma L, Coll M, Badia J, Vega MC: Overproduction, crystallization and preliminary $X$-ray analysis of the putative L-ascorbate-6-phosphate lactonase UlaG from Escherichia coli. Acta Crystallogr Sect F Struct Biol Cryst Commun 2008, 64(Pt 1):36-38

20. Campos E, Baldoma L, Aguilar J, Badia J: Regulation of expression of the divergent ulaG and ulaABCDEF operons involved in LaAscorbate dissimilation in Escherichia coli. J Bacteriol 2004, 186(6):1720-1728.

21. Garces F, Fernandez FJ, Gomez AM, Perez-Luque R, Campos E, Prohens R, Aguilar J, Baldoma L, Coll M, Badia J, et al: Quaternary structural transitions in the DeoR-type repressor UlaR control transcriptional readout from the L-ascorbate utilization regulon in Escherichia coli. Biochemistry 2008, 47(44):11424-11433.

22. Price MN, Arkin AP, Alm EJ: The life-cycle of operons. PLoS Genet 2006, 2(6):e96.

23. Lerat $E$, Daubin $V$, Moran NA: From gene trees to organismal phylogeny in prokaryotes: the case of the gamma-Proteobacteria. PLoS Biol 2003, 1(1):E19.

24. Gupta RS: Protein phylogenies and signature sequences: A reappraisal of evolutionary relationships among archaebacteria, eubacteria, and eukaryotes. Microbiol Mol Biol Rev 1998, 62(4):1435-1491.

25. Aravind $L$ : An evolutionary classification of the metallo-beta-lactamase fold proteins. In Silico Biol 1999, 1(2):69-91.

26. Moreno-Hagelsieb G, Trevino V, Perez-Rueda E, Smith TF, Collado-Vides J: Transcription unit conservation in the three domains of life: a perspective from Escherichia coli. Trends Genet 2001, 17(4):175-177.
27. Harmon-Smith M, Celia L, Chertkov O, Lapidus A, Copeland A, Glavina Del Rio T, Nolan M, Lucas S, Tice H, Cheng JF, et al: Complete genome sequence of Sebaldella termitidis type strain (NCTC 11300). Stand Genomic Sci 2010, 2(2):220-227.

28. Hubner S, Declerck N, Diethmaier C, Le Coq D, Aymerich S, Stulke J: Prevention of cross-talk in conserved regulatory systems: identification of specificity determinants in RNA-binding anti-termination proteins of the BglG family. Nucleic acids research 2011.

29. Demuth JP, Hahn MW: The life and death of gene families. Bioessays 2009, 31(1):29-39

30. Krissinel E, Henrick K: Secondary-structure matching (SSM), a new tool for fast protein structure alignment in three dimensions. Acta Crystallogr D Biol Crystallogr 2004, 60(Pt 12 Pt 1):2256-2268.

31. Mandel CR, Kaneko S, Zhang H, Gebauer D, Vethantham V, Manley JL, Tong L: Polyadenylation factor CPSF-73 is the pre-mRNA 3'-endprocessing endonuclease. Nature 2006, 444(7121):953-956.

32. de la Sierra-Gallay IL, Pellegrini O, Condon C: Structural basis for substrate binding, cleavage and allostery in the tRNA maturase RNase Z. Nature 2005, 433(7026):657-661.

33. Eddy SR: A probabilistic model of local sequence alignment that simplifies statistical significance estimation. PLoS computational biology 2008, 4(5):e1000069.

34. Waldron KJ, Robinson NJ: How do bacterial cells ensure that metalloproteins get the correct metal? Nat Rev Microbiol 2009, 7(1):25-35

35. consortium U: The Universal Protein Resource (UniProt) in 2010. Nucleic Acids Res 2010, 38(Database issue):D142-148.

36. Rajewsky N, Socci ND, Zapotocky M, Siggia ED: The evolution of DNA regulatory regions for proteo-gamma bacteria by interspecies comparisons. Genome Res 2002, 12(2):298-308.

37. Teichmann SA, Babu MM: Gene regulatory network growth by duplication. Nat Genet 2004, 36(5):492-496.

38. Lozada-Chavez I, Janga SC, Collado-Vides J: Bacterial regulatory networks are extremely flexible in evolution. Nucleic Acids Res 2006, 34(12):3434-3445.

39. Altschul SF, Madden TL, Schaffer AA, Zhang J, Zhang Z, Miller W, Lipman DJ: Gapped BLAST and PSI-BLAST: a new generation of protein database search programs. Nucleic Acids Res 1997, 25(17):3389-3402.

40. Larkin MA, Blackshields G, Brown NP, Chenna R, McGettigan PA, McWilliam H, Valentin F, Wallace IM, Wilm A, Lopez R, et al: Clustal W and Clustal X version 2.0. Bioinformatics 2007, 23(21):2947-2948.

41. Edgar RC: MUSCLE: multiple sequence alignment with high accuracy and high throughput. Nucleic Acids Res 2004, 32(5):1792-1797.

42. Castresana J: Selection of conserved blocks from multiple alignments for their use in phylogenetic analysis. Mol Biol Evol 2000, 17(4):540-552.

43. Stamatakis A: RAxML-VI-HPC: maximum likelihood-based phylogenetic analyses with thousands of taxa and mixed models. Bioinformatics 2006, 22(21):2688-2690.

44. Ronquist F, Huelsenbeck JP: MrBayes 3: Bayesian phylogenetic inference under mixed models. Bioinformatics 2003, 19(12):1572-1574.

45. Whelan S, Goldman N: A general empirical model of protein evolution derived from multiple protein families using a maximum-likelihood approach. Mol Biol Evol 2001, 18(5):691-699.

46. Yang Z: Maximum-likelihood estimation of phylogeny from DNA sequences when substitution rates differ over sites. Mol Biol Evol 1993, 10(6):1396-1401.

47. Schmidt HA, Strimmer K, Vingron M, von Haeseler A: TREE-PUZZLE: maximum likelihood phylogenetic analysis using quartets and parallel computing. Bioinformatics 2002, 18(3):502-504.

48. Shimodaira $\mathrm{H}$, Hasegawa M: Multiple comparisons of log-likelihoods with applications to phylogenetic interference. Mol Biol Evol 1999, 16:1114-1116.

49. Maglott D, Ostell J, Pruitt KD, Tatusova T: Entrez Gene: gene-centered information at NCBI. Nucleic Acids Res 2007, 35(Database issue):D26-31.

50. Eswar N, Eramian D, Webb B, Shen MY, Sali A: Protein structure modeling with MODELLER. Methods Mol Biol 2008, 426:145-159.

51. Kaufmann KW, Lemmon GH, Deluca SL, Sheehan JH, Meiler J: Practically useful: what the Rosetta protein modeling suite can do for you. Biochemistry 2010, 49(14):2987-2998.

52. Kim DE, Chivian D, Baker D: Protein structure prediction and analysis using the Robetta server. Nucleic Acids Res 2004, 32(Web Server issue): W526-531. 
53. Krivov GG, Shapovalov MV, Dunbrack RL Jr: Improved prediction of protein side-chain conformations with SCWRL4. Proteins 2009, 77(4):778-795.

54. Davis IW, Leaver-Fay A, Chen VB, Block JN, Kapral GJ, Wang X, Murray LW, Arendall WB, Snoeyink J, Richardson JS, et al: MolProbity: all-atom contacts and structure validation for proteins and nucleic acids. Nucleic acids research 2007, 35(Web Server issue):W375-383.

55. Davis FP, Braberg H, Shen MY, Pieper U, Sali A, Madhusudhan MS: Protein complex compositions predicted by structural similarity. Nucleic acids research 2006, 34(10):2943-2952.

56. Krissinel E, Henrick K: Inference of macromolecular assemblies from crystalline state. J Mol Bio/ 2007, 372(3):774-797.

doi:10.1186/1471-2148-11-273

Cite this article as: Fernandez et al:: The UlaG protein family defines novel structural and functional motifs grafted on an ancient RNase fold. BMC Evolutionary Biology 2011 11:273.

\section{Submit your next manuscript to BioMed Central} and take full advantage of:

- Convenient online submission

- Thorough peer review

- No space constraints or color figure charges

- Immediate publication on acceptance

- Inclusion in PubMed, CAS, Scopus and Google Scholar

- Research which is freely available for redistribution

Submit your manuscript at www.biomedcentral.com/submit 